\title{
Virtual Eigenvalues of the High Order Schrödinger Operator I
}

\author{
Jonathan Arazy \\ Leonid Zelenko
}

Vienna, Preprint ESI 1705 (2005)

October 3, 2005

Supported by the Austrian Federal Ministry of Education, Science and Culture Available via http://www.esi.ac.at 


\title{
Virtual eigenvalues of the high order Schrödinger operator I
}

\author{
Jonathan Arazy and Leonid Zelenko *
}

\begin{abstract}
We consider the Schrödinger operator $H_{\gamma}=(-\Delta)^{l}+\gamma V(\mathbf{x})$. acting in the space $L_{2}\left(\mathbb{R}^{d}\right)$, where $2 l \geq d, V(\mathbf{x}) \geq 0, V(\mathbf{x})$ is continuous and is not identically zero, and $\lim _{|\mathbf{x}| \rightarrow \infty} V(\mathbf{x})=0$. We obtain an asymptotic expansion as $\gamma \uparrow 0$ of the bottom negative eigenvalue of $H_{\gamma}$, which is born at the moment $\gamma=0$ from the lower bound $\lambda=0$ of the spectrum $\sigma\left(H_{0}\right)$ of the unperturbed operator $H_{0}=(-\Delta)^{l}$ (a virtual eigenvalue). To this end we develop a supplement to the Birman-Schwinger theory on the process of the birth of eigenvalues in the gap of the spectrum of the unperturbed operator $H_{0}$. Furthermore, we extract a finite-rank portion $\Phi(\lambda)$ from the Birman-Schwinger operator $X_{V}(\lambda)=V^{\frac{1}{2}} R_{\lambda}\left(H_{0}\right) V^{\frac{1}{2}}$, which yields the leading terms for the desired asymptotic expansion.
\end{abstract}

Mathematics Subject Classification 2000, Primary: 47F05, Secondary: 47E05, $35 \mathrm{Pxx}$

Keywords. Schrödinger operator, virtual eigenvalues, coupling constant, asymptotic behavior of virtual eigenvalues, Birman-Schwinger principle

\section{Introduction}

In the present work, divided into two parts (part I is the present paper, and part II is $[\mathrm{Ar}-\mathrm{Z} 2])$, we consider the elliptic differential operator of order $2 l(l \in \mathbb{N})$

$$
H_{\gamma}=(-\Delta)^{l}+\gamma V(\mathbf{x})
$$

acting in the space $L_{2}\left(\mathbb{R}^{d}\right)$. Here $V(\mathbf{x})$. is the multiplication operator in $L_{2}\left(\mathbb{R}^{d}\right)$ by the continuous, real-valued function $V(\mathbf{x})$ on $\mathbb{R}^{d}$, which is assumed to be nonnegative, not identically zero, and tends to zero sufficiently fast as $|\mathbf{x}| \rightarrow \infty$. We shall denote this operator briefly by $V$. We assume that the "coupling constant" $\gamma$ is real. As it is conventional in the literature, we call the operator $H_{\gamma}$ the Schrödinger operator of order $2 l$ and we call the function $V(\mathbf{x})$ the potential.

We consider the so-called virtual eigenvalues of the operator $H_{\gamma}$. These are the negative eigenvalues which are born at the moment $\gamma=0$ from the endpoint $\lambda=0$

${ }^{*}$ Both authors were partially supported from the Israel Science Foundation (ISF), grant number $585 / 00$, and from the German-Israeli Foundation (GIF), grant number I-619-17.6/2001. The second author was partially supported also by the KAMEA Project for Scientific Absorption in Israel. 
of the gap $(-\infty, 0)$ of the spectrum $\sigma\left(H_{0}\right)$ of the unperturbed operator $H_{0}=(-\Delta)^{l}$, while $\gamma$ varies from 0 to a small negative value $\gamma_{0}$ (see Definitions 3.14 and 3.13). Notice that in the literature the asymptotic behavior of the discrete negative spectrum of the operator $H_{\gamma}$ is well studied for $|\gamma| \rightarrow \infty$ ([Bi2], [Bi-So], [Sob]). In the paper of M. Sh. Birman [Bi1] (1961) a variational approach has been worked out for the study of birth of eigenvalues in the gap of the continuous spectrum for a small potential $V(\mathbf{x})$. In the 1970's the asymptotic behavior of virtual eigenvalues of the Schrödinger operator as $\gamma \uparrow 0$ was studied in the cases $l=1$ and $d=1,2,3$ with the help of analytical methods ([Re-Si], [S], [S1], [Kl], [B-G-S]). These investigations were based on the so-called Birman-Schwinger principle, which describes a behavior of the discrete spectrum of the perturbed operator in the gaps of the spectrum of the unperturbed one ([Bi3], [Sc], [Re-Si], [S]).

The interest in this subject was renewed in the last decade. In [W] T. Weidl has developed the Birman approach for the study of the existence of virtual eigenvalues for a wide class of elliptic differential operators of high order and even for indefinite perturbations. In particular (see [W], Corollary 6.1): if $2 l \geq d$, the potential $V(\mathbf{x})$ is continuous, non-negative, not identically zero and $V(\mathbf{x}) \rightarrow 0$ as $|\mathbf{x}| \rightarrow \infty$ sufficiently fast, then the operator $H_{\gamma}$ of the form (1.1) has exactly $r=\left(\begin{array}{c}m+d \\ d\end{array}\right)$ virtual eigenvalues at the point $\lambda=0$, where $m=l-\left[\frac{d+1}{2}\right]$.

The paper $[\mathrm{N}-\mathrm{W}]$ is devoted to a generalization of Lieb-Thirring inequalities (obtained in [L-Thr] for the Schrödinger operator of second order) to the operator $H_{\gamma}$ of a high order $2 l$. Furthermore, an asymptotic representation as $\gamma \uparrow 0$ for the bottom virtual eigenvalue of the operator $H_{\gamma}$ has been obtained in [N-W], in which the leading coefficient has been evaluated explicitly ([N-W], Lemma 5.1).

In the present paper we obtain asymptotic expansion for the bottom virtual eigenvalue of the operator $H_{\gamma}$ as $\gamma \uparrow 0$, which is more precise than the corresponding asymptotic formula in $[\mathrm{N}-\mathrm{W}]$ (see Remark 5.8). In [Ar-Z2] we obtain asymptotic estimates for non-bottom virtual eigenvalues.

Unlike the variational approach of $[\mathrm{W}]$, we use an analytical method in combination with some variational arguments. The idea behind this approach follows B. Simon's paper [S1] (see also [Re-Si] and [S]). Studying the Birman-Schwinger operator

$$
X_{V}(\lambda)=V^{\frac{1}{2}}\left((-\Delta)^{l}-\lambda I\right)^{-1} V^{\frac{1}{2}}(\lambda<0),
$$

we extract a finite-rank portion from the kernel

$$
\frac{1}{(2 \pi)^{d}} \int_{\mathbb{R}^{d}} \frac{\exp (i \mathbf{p} \cdot(\mathbf{x}-\mathbf{y})) d \mathbf{p}}{|\mathbf{p}|^{2 l}+|\lambda|} .
$$

of the resolvent $\left((-\Delta)^{l}-\lambda I\right)^{-1}$. We obtain this finite-rank portion as corresponding to first terms of the Taylor expansion of the exponent at $\mathbf{p}=\mathbf{0}$. We choose the number of these terms such that for the corresponding finite-rank portion $\Phi(\lambda)$ of the Birman-Schwinger operator $X_{V}(\lambda)$ the norm of the remainder $X_{V}(\lambda)-\Phi(\lambda)$ is uniformly bounded with respect to $\lambda$ in $(-\delta, 0)$ for some $\delta>0$. Afterwards we obtain asymptotic expansions, with respect to a small $\lambda<0$, of eigenvalues of the operator $\Phi(\lambda)$ which grow to $+\infty$ as $\lambda \uparrow 0$. The inversion of these asymptotic expansions yields the leading terms of the desired asymptotic representation of the virtual eigenvalues of the operator $H_{\gamma}$ for $\gamma \uparrow 0$. Unlike our approach, in $[\mathrm{N}-\mathrm{W}]$ for the Schrödinger operator of high order only rank-one portion has been extracted from the operator $X_{V}(\lambda)$. 
Hence, in general the corresponding remainder does not have there the property mentioned above. This advantage of our approach enables us to obtain an asymptotic estimate for the bottom virtual eigenvalue of the operator $H_{\gamma}$, which is more precise than the estimate obtained in $[\mathrm{N}-\mathrm{W}]$. This approach also enables us to get asymptotic estimates for the non-bottom virtual eigenvalues in Part II of the work.

The paper is divided into five sections. After this introduction (Section 1), we give in Section 2 the list of notation used in the paper.

Section 3 is devoted to a supplement to the Birman-Schwinger theory, in which we study the process of the birth of eigenvalues in a gap of the spectrum of the unperturbed operator for a small coupling constant. This is a generalization (to the case of relatively compact perturbations) of the theory developed in our earlier paper [Ar-Z] for the case of finite-rank perturbations. The concept of main characteristic branches of an operator $H_{0}$ with respect to a perturbing operator $V$ plays a pivotal role in these considerations (see Definition 3.9 and Definition 3.2). It enables us to get asymptotic estimates of virtual eigenvalues of the operator $H_{\gamma}$ for $\gamma \uparrow 0$.

Section 4 is devoted to the above mentioned extraction of a finite-rank portion $\Phi(\lambda)$ from the Birman-Schwinger operator $X_{V}(\lambda)$ (see Propositions 4.4 and 4.8).

In Section 5 we obtain asymptotic expansion for the bottom virtual eigenvalue of the operator $H_{\gamma}$ with respect to a small coupling constant $\gamma<0$ (Theorems 5.3 and 5.6). To this end we carry out an asymptotic expansion for the maximal eigenvalue $\tilde{\mu}_{0}(\lambda)$ of the finite-rank portion $\Phi(\lambda)$ of the Birman-Schwinger operator $X_{V}(\lambda)$. In the case of $\mathbb{R}^{d}$ with $d$ odd $\tilde{\mu}_{0}\left(-t^{2 l}\right)$ admits expansion in rational Laurent series near the point $t=0$, because the operator function $\Phi\left(-t^{2 l}\right)$ is meromorphic (Lemma 5.1). Hence the asymptotic expansion of the bottom virtual eigenvalue has a power form in the case of an odd $d$ (Theorem 5.3). The expansion of $\tilde{\mu}_{0}\left(-t^{2 l}\right)$ can be easily derived with the help of a simple version of the Schrödinger method ([Bau], Ch. 3, $n^{o} 3.1 .2$ ) thanks the fact that the quantity $t^{2 l-d} \tilde{\mu}_{0}\left(-t^{2 l}\right)$ is born at the moment $t=0$ from a simple eigenvalue of the operator

$$
\Phi_{0}=\lim _{t \downarrow 0} t^{2 l-d} \Phi\left(-t^{2 l}\right)
$$

(see Lemma 5.1). In the case of $d$ even the operator function $\Phi\left(-t^{2 l}\right)$ is not meromorphic, because it contains summands with $\ln \left(\frac{1}{t}\right)$ in its expansion near the point $t=0$. Hence, the asymptotic expansion of the bottom virtual eigenvalue is more complicated for an even $d$. We use in this case a modification of the method mentioned above (see Lemma 5.4 and Theorem 5.6).

\section{Notation}

In this section we give a list of notation used in the present paper.

$\mathbb{Z}$ is the ring of all integers;

$\mathbb{I N}$ is the set of all natural numbers $1,2, \ldots$;

$\mathbb{Z}_{+}=\mathbb{N} \cup\{0\}$;

$\mathbb{R}$ is the field of all real numbers;

$\mathbb{R}_{+}=[0, \infty)$;

$\mathbb{C}$ is the field of all complex numbers;

$\Re(z), \Im(z)$ are the real and the imaginary parts of a number $z \in \mathbb{C}$. 
$\# S$ is the number of elements of a finite set $S$.

$\mathcal{O}(x)$ is generic notation for a neighborhood of a point $x$;

$\operatorname{cl}(\mathcal{S})$ is the closure of a set $\mathcal{S}$.

If $M$ is a metric space, then $\operatorname{dist}(x, y)$ and $\operatorname{dist}(x, Y)$ are the distance between points $x, y \in M$ and the distance between a point $x \in M$ and a set $Y \subseteq M$.

$\mathbb{C}^{d}=\times_{j=1}^{d} \mathbb{C} ; \quad \mathbb{R}^{d}=\times_{j=1}^{d} \mathbb{R} ; \quad \mathbb{Z}_{+}^{d}=\times_{j=1}^{d} \mathbb{Z}_{+}$.

$\mathbf{x} \cdot \mathbf{y}=\sum_{j=1}^{d} x_{j} y_{j}$ is the canonical inner product of vectors $\mathbf{x}=\left(x_{1}, x_{2}, \ldots, x_{d}\right)$

and $\mathbf{y}=\left(y_{1}, y_{2}, \ldots, y_{d}\right)$ belonging to $\mathbb{R}^{d}$;

$|\mathbf{x}|=\sqrt{\mathbf{x} \cdot \mathbf{x}}$ is the Euclidean norm in $\mathbb{R}^{d}$;

$|\mathbf{k}|=\sum_{j=1}^{d}\left|k_{j}\right|$ is the $l_{1}$-norm of a multi-index $\mathbf{k}=\left(k_{1}, k_{2}, \ldots, k_{d}\right) \in \mathbb{Z}_{+}^{d}$.

$\sigma_{d}$ is the $d$-1-dimensional volume measure of the unit sphere

$$
\left\{\mathbf{x} \in \mathbb{R}^{d}|| \mathbf{x} \mid=1\right\} .
$$

$\mathbf{x}^{\mathbf{k}}=\prod_{j=1}^{d} x_{j}^{k_{j}}$, where $\mathbf{x}=\left(x_{1}, \ldots, x_{d}\right) \in \mathbb{R}^{d}$ and $\mathbf{k}=\left(k_{1}, \ldots, k_{d}\right) \in \mathbb{Z}_{+}^{d} ;$

$\mathbf{k} !=\prod_{j=1}^{d} k_{j}$ !, where $\mathbf{k}=\left(k_{1}, \ldots, k_{d}\right) \in \mathbb{Z}_{+}^{d}$.

Let $f: \mathcal{A}_{1} \rightarrow \mathcal{A}_{2}$ be a mapping. We denote:

$\operatorname{Dom}(f)=\mathcal{A}_{1}$ is the domain of $f$;

$\operatorname{Im}(f)=f\left(\mathcal{A}_{1}\right)$ is the image (range) of $f$;

$\left.f\right|_{\mathcal{G}}$ is the restriction of $f$ on a subset $\mathcal{G} \subset \mathcal{A}_{1}$.

If $A$ is a closed linear operator acting in a Hilbert space $\mathcal{H}$, then:

$\operatorname{ker}(A)$ is the kernel of $A$;

$\mathcal{R}(A)$ is the resolvent set of $A$, that is the set of all $\lambda \in \mathbb{C}$ such that $A-\lambda I$

is continuously invertible;

$R_{\lambda}(A) \quad(\lambda \in \mathcal{R}(A))$ is the resolvent of $A$, that is $R_{\lambda}(A)=(A-\lambda I)^{-1}$;

$\sigma(A)=\mathbb{C} \backslash \mathcal{R}(A)$ is the spectrum of $A$.

$P_{G}$ is the orthogonal projection on a closed subspace $G$ of a Hilbert space $\mathcal{H}$.

$\mathcal{S}_{2}$ is the Hilbert-Schmidt class of operators acting in a Hilbert space $\mathcal{H}$;

$\|T\|_{2}$ is the Hilbert-Schmidt norm of the operator $T \in \mathcal{S}_{2}$.

$\hat{f}(\mathbf{p})=(2 \pi)^{-\frac{d}{2}} \int_{\mathbb{R}^{d}} f(\mathbf{x}) e^{-i \mathbf{x} \cdot \mathbf{p}} d \mathbf{x}$ is the Fourier transform of a function $f$;

$(f \star g)(\mathbf{x})=(2 \pi)^{-\frac{d}{2}} \int_{\mathbb{R}^{d}} f(\mathbf{x}-\mathbf{y}) g(\mathbf{y}) d \mathbf{y}$ is the convolution of $f$ and $g$.

For a power series $p(\epsilon)=\sum_{k=0}^{\infty} p_{k} \epsilon^{k}$ and $j \in \mathbb{Z}_{+}$we denote

$$
(p(\epsilon))_{j}=\sum_{k=0}^{j} p_{k} \epsilon^{k} .
$$

\section{General results on the birth of eigenvalues in a gap of the unperturbed spectrum}

Let $H_{0}, V$ be self-adjoint operators acting in a Hilbert space $\mathcal{H}$, such that $D=$ $\operatorname{Dom}\left(H_{0}\right) \subseteq \operatorname{Dom}(V)$ and $D$ is dense in $\mathcal{H}$. For $\gamma \in \mathbb{R}$ (a "coupling constant") 
consider the perturbed operator

$$
H_{\gamma}=H_{0}+\gamma V \text {. }
$$

In this section we shall assume that the following conditions are satisfied:

(A) $(a, b)(-\infty \leq a<b \leq+\infty)$ is a gap of the spectrum $\sigma\left(H_{0}\right)$ of the unperturbed operator $H_{0}$.

(B) The operator $V$ is bounded ${ }^{1}, V \geq 0$ and for some $\lambda_{0} \in(a, b)$ the operator $V^{\frac{1}{2}} R_{\lambda_{0}}\left(H_{0}\right)$ is compact.

The unperturbed operator $H_{0}$ may be unbounded.

The theory developed in this section answers the following questions: how many eigenvalues of the operator $H_{\gamma}$ are born in the gap $(a, b)$ at the moment $\gamma=0$ and what is the rate of this birth with respect to a small $\gamma$ ?

3.1 ${ }^{\mathrm{o}}$. We shall use the following well known statement concerning the structure of the set

$$
\sigma_{\gamma}(a, b)=\sigma\left(H_{\gamma}\right) \cap(a, b)
$$

(see, for instance, [Bi2], Proposition 1.5):

Proposition 3.1. The set $\sigma_{\gamma}(a, b)$ consists of at most countable number of eigenvalues of finite multiplicities of the operator $\mathrm{H}_{\gamma}$ and these eigenvalues can cluster only to endpoints of the gap $(a, b)$. Furthermore, this set coincides with the spectrum $\sigma(\Phi(\gamma))$ of the pencil of operators

$$
\Phi(\gamma)=\left\{I+\gamma X_{V}(\lambda)\right\}_{\lambda \in(a, b)},
$$

where

$$
X_{V}(\lambda)=V^{\frac{1}{2}} R_{\lambda}\left(H_{0}\right) V^{\frac{1}{2}} .
$$

Moreover, the operator function $X_{V}(\lambda)$ is holomorphic in $\mathcal{R}\left(H_{0}\right)$ in the operator norm, each of the operators $X_{V}(\lambda)$ is compact and any point $\lambda \in \sigma_{\gamma}(a, b)$ is an eigenvalue of the pencil $\Phi(\gamma)$ such that

$$
\operatorname{dim}\left(\operatorname{ker}\left(H_{\gamma}-\lambda I\right)\right)=\operatorname{dim}\left(\operatorname{ker}\left(I+\gamma X_{V}(\lambda)\right)\right) .
$$

As it is conventional in the literature, we shall call the operator $X_{V}(\lambda)$, defined by (3.4), the Birman-Schwinger operator.

We shall investigate some properties of the operator pencil $\Phi(\gamma)$, defined by (3.3), (3.4), and define some new concepts. Since, by Proposition 3.1, each operator $X_{V}(\lambda)(\lambda \in(a, b))$ is self-adjoint and compact, its spectrum consists of at most a countable number of real eigenvalues which can cluster only to the point 0 . Furthermore, each of the non-zero eigenvalues has finite multiplicity. Let us number all the positive eigenvalues $\mu_{k}^{+}(\lambda)_{k \in \mathbb{N}}$ in the non-increasing ordering

$$
\mu_{1}^{+}(\lambda) \geq \mu_{2}^{+}(\lambda) \geq \cdots \geq \mu_{k}^{+}(\lambda) \geq \cdots
$$

and all the negative ones $\mu_{k}^{-}(\lambda)_{k \in \mathbb{N}}$ in the non-decreasing ordering

$$
\mu_{1}^{-}(\lambda) \leq \mu_{2}^{-}(\lambda) \leq \cdots \leq \mu_{k}^{-}(\lambda) \leq \cdots
$$

\footnotetext{
${ }^{1}$ Apparently, it is possible to replace this condition by a less restrictive one, but this is sufficient for our applications.
} 
(each eigenvalue is repeated according to its multiplicity). So, by such ordering we have chosen one-valued branches of eigenvalues of the operator function $X_{V}(\lambda)$. Since the positive and negative branches can "go to zero" at some points of the gap $(a, b)$, each branch has its domain which will be denoted by $\operatorname{Dom}\left(\mu_{k}^{+}\right)$and $\operatorname{Dom}\left(\mu_{k}^{-}\right)$. Let us introduce the following

Definition 3.2. We call the positive and negative branches (3.6), (3.7) of eigenvalues of the operator function $X_{V}(\lambda)$, defined by (3.4), the characteristic branches (positive and negative) of the operator $H_{0}$ with respect to the operator $V$ on a gap $(a, b)$ of $\sigma\left(H_{0}\right)$.

$\mathbf{3 . 2} \mathbf{2}^{\mathbf{o}}$. Before studying some properties of the characteristic branches, we shall prove two lemmas which are versions of the comparison theorem for eigenvalues of selfadjoint compact operators, based on the minimax characterization of the eigenvalues ([Ri-Nag], Ch. VI, Sect. 1).

Lemma 3.3. Let $A_{1}, A_{2}$ be self-adjoint compact operators acting in a Hilbert space $\mathcal{H}$, such that

$$
A_{1}<A_{2},^{2}
$$

and

$$
\mu_{k, j}^{+} \quad\left(j=1,2, k=1,2, \ldots, N_{j}^{+}\right), \quad \mu_{k, j}^{-} \quad\left(j=1,2, k=1,2, \ldots, N_{j}^{-}\right)
$$

be the positive and negative eigenvalues of the operator $A_{j}(j=1,2)$ arranged by the non-increasing ordering of their absolute values (multiplicity counted). Then the following inequalities are valid:

$$
\begin{gathered}
N_{2}^{+} \geq N_{1}^{+}, \\
N_{2}^{-} \leq N_{1}^{-}, \\
\mu_{k, 1}^{+}<\mu_{k, 2}^{+} \quad\left(k=1,2, \ldots, N_{1}^{+}\right), \\
\mu_{k, 2}^{-}<\mu_{k, 1}^{-} \quad\left(k=1,2, \ldots, N_{2}^{-}\right) .
\end{gathered}
$$

Proof. We shall consider only the positive eigenvalues, because for the negative ones the proof is analogous. By the condition of the lemma,

$$
\left(A_{1} x, x\right)<\left(A_{2} x, x\right) \quad \forall x \in \mathcal{H}, x \neq 0 .
$$

By the minimax characterization of eigenvalues ([Ri-Nag], Ch. VI, Sect. 1, $n^{o}$ 95),

$$
\mu_{k, j}^{+}=\min _{L_{k-1} \in \mathcal{L}_{k-1}} \max _{x \in L \frac{\perp}{k-1},\|x\|=1}\left(A_{j} x, x\right) \quad(j=1,2),
$$

where $\mathcal{L}_{k}$ is the set of all $k$-dimensional subspaces of $\mathcal{H}$. Let us take $1 \leq k \leq N_{2}^{+}$. Then for some $L_{k-1} \in \mathcal{L}_{k-1}$

$$
\mu_{k, 2}^{+}=\max _{x \in L_{k-1}^{\perp},\|x\|=1}\left(A_{2} x, x\right) .
$$

\footnotetext{
${ }^{2}$ In the well known version of the comparison theorem the weak inequality $\leq$ is used, and accordingly one obtains the weak inequality for eigenvalues.
} 
Hence, if for $k \in\left\{1,2, \ldots, N_{2}^{+}\right\}$there exists $\mu_{k, 1}^{+}$, we obtain from (3.13), taking into account inequality (3.12), that

$$
\begin{aligned}
& \mu_{k, 1}^{+} \leq \max _{x \in L_{\frac{1}{k-1}},\|x\|=1}\left(A_{1} x, x\right)< \\
& <\max _{x \in L_{\frac{1}{k}-1},\|x\|=1}\left(A_{2} x, x\right)=\mu_{k, 2}^{+},
\end{aligned}
$$

i.e, we have obtained the inequalities (3.9), (3.11).

Lemma 3.4. Let $A_{1}, A_{2}$ be self-adjoint compact operators acting in a Hilbert space $\mathcal{H}$, such that for some $\epsilon>0$

$$
A_{2}-\epsilon I \leq A_{1} \leq A_{2}+\epsilon I
$$

Then for the positive and negative eigenvalues (3.8) of the operators $A_{1}$ and $A_{2}$ the following properties are valid ${ }^{3}$ :

(a) if for $k \in\left\{1,2, \ldots, N_{2}^{+}\right\}$there exists $\mu_{k, 1}^{+}$, then

$$
\mu_{k, 1}^{+} \leq \mu_{k, 2}^{+}+\epsilon ;
$$

(b) if for $k \in\left\{1,2, \ldots, N_{1}^{+}\right\}$there exists $\mu_{k, 2}^{+}$, then

$$
\mu_{k, 2}^{+} \leq \mu_{k, 1}^{+}+\epsilon
$$

(c) if for $k \in\left\{1,2, \ldots, N_{2}^{-}\right\}$there exists $\mu_{k, 1}^{-}$, then

$$
\mu_{k, 2}^{-} \leq \mu_{k, 1}^{-}+\epsilon
$$

(d) if for $k \in\left\{1,2, \ldots, N_{1}^{-}\right\}$there exists $\mu_{k, 2}^{-}$, then

$$
\mu_{k, 1}^{-} \leq \mu_{k, 2}^{-}+\epsilon \text {. }
$$

Proof. Assume that $k \in\left\{1,2, \ldots, N_{1}^{+}\right\}$. Making use of the minimax characterization of eigenvalues, we choose a subspace $L_{k-1} \in \mathcal{L}_{k-1}$ such that (3.14) is valid and, furthermore, in view of (3.15),

$$
\mu_{k, 1}^{+} \leq \max _{x \in L_{\frac{1}{-1}}^{+},\|x\|=1}\left(A_{1} x, x\right)<\max _{x \in L_{k-1}^{\perp},\|x\|=1}\left(A_{2} x, x\right)+\epsilon=\mu_{k, 2}^{+}+\epsilon .
$$

So, property (a) is proven. Property (b) follows from (a) by the interchange of $A_{1}$ and $A_{2}$ and properties (c) and (d) follow from (a) and (b) applied to the operators $-A_{1}$ and $-A_{2}$. The lemma is proven.

3.3 ${ }^{\circ}$. We now turn to properties of the characteristic branches. They are based on the following

\footnotetext{
${ }^{3}$ Here we cannot use immediately the comparison theorem, because $A_{2}-\epsilon I, A_{2}+\epsilon I$ are not compact operators
} 
Lemma 3.5. For any $\lambda \in(a, b)$ the subspace

$$
\mathcal{H}_{V}=\mathcal{H} \ominus \operatorname{ker}(V) .
$$

is invariant under the operator $X_{V}(\lambda)$, defined by (3.4), and

$$
\sigma\left(X_{V}(\lambda)\right)= \begin{cases}\sigma\left(\tilde{X}_{V}(\lambda)\right), & \text { if } \operatorname{ker}(V)=\{\mathbf{0}\}, \\ \sigma\left(\tilde{X}_{V}(\lambda)\right) \cup\{0\}, & \text { if } \operatorname{ker}(V) \neq\{\mathbf{0}\},\end{cases}
$$

where

$$
\tilde{X}_{V}(\lambda)=\left.X_{V}(\lambda)\right|_{\mathcal{H}_{V}}
$$

Furthermore, if the operator $\tilde{X}_{V}(\lambda)$ is considered as acting in the space $\mathcal{H}_{V}$, then the corresponding operator function increases on $(a, b)$, that is

$$
\lambda_{1}<\lambda_{2} \text { implies } \tilde{X}_{V}\left(\lambda_{1}\right)<\tilde{X}_{V}\left(\lambda_{2}\right) \quad \forall \lambda_{1}, \lambda_{2} \in(a, b) .
$$

Proof. As it is known,

$$
\operatorname{ker}\left(V^{\frac{1}{2}}\right)=\operatorname{ker}(V)
$$

that is

$$
\operatorname{cl}\left(\operatorname{Im}\left(V^{\frac{1}{2}}\right)\right)=\left(\operatorname{ker}\left(V^{\frac{1}{2}}\right)\right)^{\perp}=\operatorname{cl}(\operatorname{Im}(V))=\mathcal{H}_{V} .
$$

From the latter fact and (3.4) we see that $\operatorname{Im}\left(X_{V}(\lambda)\right) \subseteq \mathcal{H}_{V}$, hence the subspace $\mathcal{H}_{V}$ is invariant for the operator $X_{V}(\lambda)$. Furthermore, we see from (3.4), (3.20) that $\operatorname{ker}(V) \subseteq \operatorname{ker}\left(X_{V}(\lambda)\right)$. Since the operator $X_{V}(\lambda)$ is self-adjoint, we obtain from the above circumstances and from (3.16) that (3.17) is valid. Let us prove property (3.19). Observe that, by Proposition 3.1,

$$
X_{V}(\cdot) \in C^{1}\left((a, b), \mathcal{B}\left(\mathcal{H}_{V}\right)\right)
$$

In view of (3.4), we obtain:

$$
\frac{d}{d \lambda} X_{V}(\lambda)=V^{\frac{1}{2}}\left(R_{\lambda}\left(H_{0}\right)\right)^{2} V^{\frac{1}{2}} .
$$

Then for any $x \in \mathcal{H}_{V}, x \neq 0, \lambda \in(a, b)$

$$
\frac{d}{d \lambda}\left(\tilde{X}_{V}(\lambda) x, x\right)=\left(R_{\lambda}\left(H_{0}\right) V^{\frac{1}{2}} x, R_{\lambda}\left(H_{0}\right) V^{\frac{1}{2}} x\right)>0,
$$

because $\operatorname{ker}\left(R_{\lambda}\left(H_{0}\right)\right)=\{0\}$ and, in view of (3.20) and (3.16), $V^{\frac{1}{2}} x \neq 0$. The latter inequality implies (3.19).

Remark 3.6. In view of (3.17), the union of the sequences (3.6) and (3.7) coincides with the set of the non-zero eigenvalues of the operator $\tilde{X}_{V}(\lambda)$ defined by (3.18).

The following properties of the characteristic branches (Definition 3.2) are valid:

Proposition 3.7. All the positive and negative characteristic branches $\mu_{k}^{+}(\lambda), \mu_{k}^{-}(\lambda)$ of $H_{0}$, with respect to $V$ on the gap $(a, b)$, are continuous and increasing on their domains, these domains have the form

$$
\operatorname{Dom}\left(\mu_{k}^{+}\right)=\left(\eta_{k}^{+}, b\right), \eta_{k}^{+} \in[a, b],
$$




$$
\operatorname{Dom}\left(\mu_{k}^{-}\right)=\left(a, \eta_{k}^{-}\right), \eta_{k}^{-} \in[a, b] .
$$

and the following property is valid:

$$
\begin{aligned}
& \eta_{k}^{+} \in(a, b) \Rightarrow \lim _{\lambda \downarrow \eta_{k}^{+}} \mu_{k}^{+}(\lambda)=0, \\
& \eta_{k}^{-} \in(a, b) \Rightarrow \lim _{\lambda \uparrow \eta_{k}^{-}} \mu_{k}^{-}(\lambda)=0 .
\end{aligned}
$$

Furthermore, the sequence $\left\{\eta_{k}^{+}\right\}$is non-decreasing and the sequence $\left\{\eta_{k}^{-}\right\}$is nonincreasing.

Proof. By (3.19), Lemma 3.3 and Remark 3.6, if $\lambda_{1}, \lambda_{2} \in(a, b), \lambda_{1}<\lambda_{2}$ and there exists $\mu_{k}^{+}\left(\lambda_{1}\right)$, then there exists $\mu_{k}^{+}\left(\lambda_{2}\right)$ and

$$
\mu_{k}^{+}\left(\lambda_{1}\right)<\mu_{k}^{+}\left(\lambda_{2}\right)
$$

This means that either (3.22) or

$$
\operatorname{Dom}\left(\mu_{k}^{+}\right)=\left[\eta_{k}^{+}, b\right), \eta_{k}^{+} \in(a, b]
$$

is valid, and, furthermore, the function $\mu_{k}^{+}(\lambda)$ increases on $\operatorname{Dom}\left(\mu_{k}^{+}\right)$. Let us show that only the case (3.22) is realized. Take $\lambda_{0} \in \operatorname{Dom}\left(\mu_{k}^{+}\right)$. In view of (3.21), for any $\epsilon \in\left(0, \mu_{k}^{+}\left(\lambda_{0}\right)\right)$ there exists $\delta>0$, such that $\left(\lambda_{0}-\delta, \lambda_{0}+\delta\right) \subset(a, b)$ and

$$
X_{V}\left(\lambda_{0}\right)-\epsilon I<X_{V}(\lambda)<X_{V}\left(\lambda_{0}\right)+\epsilon I .
$$

Then, by Lemma 3.4, for any $\lambda \in\left(\lambda_{0}-\delta, \lambda_{0}+\delta\right)$ there exists $\mu_{k}^{+}(\lambda)$ and

$$
\mu_{k}^{+}\left(\lambda_{0}\right)-\epsilon<\mu_{k}^{+}(\lambda)<\mu_{k}^{+}\left(\lambda_{0}\right)+\epsilon .
$$

This means that $\left(\lambda_{0}-\delta, \lambda_{0}+\delta\right) \subset \operatorname{Dom}\left(\mu_{k}^{+}\right)$. So, we have proved that the set $\operatorname{Dom}\left(\mu_{k}^{+}\right)$ is open, that is (3.22) is valid, and, furthermore, the function $\mu_{k}^{+}(\lambda)$ is continuous on $\operatorname{Dom}\left(\mu_{k}^{+}\right)$.

Let us prove relation (3.24). Assume, on the contrary, that

$$
\mu_{k}^{+}\left(\eta_{k}^{+}+0\right)=\lim _{\lambda \downarrow \eta_{k}^{+}} \mu_{k}^{+}(\lambda)>0
$$

for $\eta_{k}^{+} \in(a, b)$. Then from the left inequality (3.26), with $\lambda_{0}=\eta_{k}^{+}$, and Lemma 3.3 we obtain that for any $\epsilon \in\left(0, \mu_{k}^{+}\left(\eta_{k}^{+}+0\right)\right)$ there exists $\delta>0$, such that $\left(\eta_{k}^{+}-\delta, \eta_{k}^{+}\right] \subset$ $\operatorname{Dom}\left(\mu_{k}^{+}\right)$. This contradicts equality (3.22). So, (3.24) is valid.

Since for any fixed $\lambda \in\left(\eta_{k}^{+}, b\right)$ the sequence $\mu_{k}^{+}(\lambda)$ is non-increasing, then $\left(\eta_{k}^{+}, b\right) \subseteq$ $\left(\eta_{j}^{+}, b\right)$ for $j<k$, that is the sequence $\eta_{k}^{+}$is non-decreasing.

The corresponding properties (3.23) and (3.25) of the negative branches $\mu_{k}^{-}(\lambda)$ are proved analogously.

Corollary 3.8. For the characteristic branches of $H_{0}$ with respect to $V$ on the gap $(a, b)$ there exist the limits (finite or infinite):

$$
\begin{aligned}
& \mu_{k, b}^{+}=\lim _{\lambda_{\uparrow} b} \mu_{k}^{+}(\lambda), \\
& \mu_{k, a}^{-}=\lim _{\lambda_{\downarrow} a} \mu_{k}^{-}(\lambda) .
\end{aligned}
$$


We can introduce the following

Definition 3.9. Consider $l(a), l(b) \in \mathbb{Z}_{+} \cup\{+\infty\}$ defined by the conditions:

$$
\begin{gathered}
-\infty=\mu_{1, a}^{-}=\mu_{2, a}^{-}=\cdots=\mu_{l(a), a}^{-}<\mu_{l(a)+1, a}^{-} \leq \mu_{l(a)+2, a}^{-} \leq \ldots, \\
+\infty=\mu_{1, b}^{+}=\mu_{2, b}^{+}=\cdots=\mu_{l(b), b}^{+}>\mu_{l(b)+1, b}^{+} \geq \mu_{l(b)+2, b}^{+} \geq \ldots,
\end{gathered}
$$

were the quantities $\mu_{k, a}^{-}, \mu_{k, b}^{+}$are defined by (3.27) and (3.28). We shall call $l(a)$ and $l(b)$ the asymptotic multiplicities of the endpoints $a$ and $b$ of the gap $(a, b)$ of $\sigma\left(H_{0}\right)$ with respect to the operator $V$ and denote them

$$
l(a)=M\left(a, H_{0}, V\right), \quad l(b)=M\left(b, H_{0}, V\right) .
$$

The characteristic branches

$$
\left\{\mu_{k}^{-}(\lambda)\right\}_{k=1}^{l(a)}, \quad\left\{\mu_{k}^{+}(\lambda)\right\}_{k=1}^{l(b)}
$$

are called the main characteristic branches of the operator $H_{0}$ with respect to the operator $V$ near the endpoints $a$ and $b$ respectively.

The following property of the main characteristic branches holds:

Proposition 3.10. If

$$
M\left(b, H_{0}, V\right)=\infty,
$$

then all the positive characteristic branches $\mu_{k}^{+}(\lambda)$ of $H_{0}$ with respect to $V$ on $(a, b)$ are the main characteristic branches near the endpoint $b$. If $M\left(a, H_{0}, V\right)=\infty$, then all the negative characteristic branches $\mu_{k}^{-}(\lambda)$ of $H_{0}$ with respect to $V$ on $(a, b)$ are the main characteristic branches near the endpoint a.

Proof. Assume, on the contrary, that a positive branch $\mu_{k_{0}}^{+}(\lambda)$ is not the main one, i.e, the quantity $\mu_{k_{0}, b}^{+}$, defined by (3.27)), is finite (see Definition 3.9). Since for any fixed $\lambda \in(a, b)$ the sequence $\mu_{k}^{+}(\lambda)$ is non-increasing, then for any $k>k_{0} \mu_{k, b}^{+}<\infty$. This contradicts the assumption (3.29). The case of the negative characteristic branches is treated analogously.

3.4. ${ }^{\mathrm{o}}$. We now turn to the study of the eigenvalues of the operator $H_{\gamma}$, defined by (3.1), which appear in the gap $(a, b)$ of $\sigma\left(H_{0}\right)$ for a small $\gamma$. First of all, we shall describe a general picture of the birth of the eigenvalues in the gap $(a, b)$.

Proposition 3.11. (i) The set $\sigma_{\gamma}(a, b)$, defined by (3.2), has the representation:

$$
\sigma_{\gamma}(a, b)= \begin{cases}\bigcup_{k=1}^{\infty}\left\{\lambda_{k}^{+}(\gamma)\right\} & \text { for } \gamma<0 \\ \bigcup_{k=1}^{\infty}\left\{\lambda_{k}^{-}(\gamma)\right\} & \text { for } \gamma>0\end{cases}
$$

where

$$
\begin{aligned}
& \lambda_{k}^{+}(\gamma)=\left(\mu_{k}^{+}\right)^{-1}\left(-\frac{1}{\gamma}\right), \\
& \lambda_{k}^{-}(\gamma)=\left(\mu_{k}^{-}\right)^{-1}\left(-\frac{1}{\gamma}\right)
\end{aligned}
$$


and $\left(\mu_{k}^{+}\right)^{-1},\left(\mu_{k}^{-}\right)^{-1}$ are the inverses of the functions $\mu_{k}^{+}(\lambda), \mu_{k}^{-}(\lambda)$, the positive and negative characteristic branches of $H_{0}$ with respect to $V$ on the gap $(a, b)$.

(ii) Each of the functions $\lambda_{k}^{+}(\gamma)$ has a domain of the form

$$
\operatorname{Dom}\left(\lambda_{k}^{+}\right)=\left(\gamma_{k}^{+}, \tilde{\gamma}_{k}^{+}\right)\left(\gamma_{k}^{+}<\tilde{\gamma}_{k}^{+} \leq 0\right)
$$

it is continuous and increasing there, and satisfies the conditions:

$$
\begin{gathered}
\lim _{\gamma \uparrow \tilde{\gamma}_{k}^{+}} \lambda_{k}^{+}(\gamma)=b \\
\lim _{\gamma \downarrow \gamma_{k}^{+}} \lambda_{k}^{+}(\gamma)=\eta_{k}^{+}
\end{gathered}
$$

(recall that $\left.\operatorname{Dom}\left(\mu_{k}^{+}\right)=\left(\eta_{k}^{+}, b\right)\right)$. Furthermore,

$$
\eta_{k}^{+} \in(a, b) \Rightarrow \gamma_{k}^{+}=-\infty
$$

and

$$
\eta_{k}^{+}=a=-\infty \Rightarrow \gamma_{k}^{+}=-\infty .
$$

The sequences $\left\{\gamma_{k}^{+}\right\},\left\{\tilde{\gamma}_{k}^{-}\right\}$are non-increasing.

(iii) In the analogous manner, each of the functions $\lambda_{k}^{-}(\gamma)$ has domain of the form

$$
\operatorname{Dom}\left(\lambda_{k}^{-}\right)=\left(\tilde{\gamma}_{k}^{-}, \gamma_{k}^{-}\right) \quad\left(0 \leq \tilde{\gamma}_{k}^{-}<\gamma_{k}^{-}\right)
$$

it is continuous and increasing there, and satisfies the conditions:

$$
\begin{gathered}
\lim _{\gamma \downarrow \tilde{\gamma}_{k}^{-}} \lambda_{k}^{-}(\gamma)=a \\
\lim _{\gamma \uparrow \gamma_{k}^{-}} \lambda_{k}^{-}(\gamma)=\eta_{k}^{-}
\end{gathered}
$$

(recall that $\left.\operatorname{Dom}\left(\mu_{k}^{-}\right)=\left(a, \eta_{k}^{-}\right)\right)$. Furthermore,

$$
\eta_{k}^{-} \in(a, b) \Rightarrow \gamma_{k}^{-}=+\infty
$$

and

$$
\eta_{k}^{-}=b=+\infty \Rightarrow \gamma_{k}^{-}=+\infty
$$

The sequences $\left\{\gamma_{k}^{-}\right\},\left\{\tilde{\gamma}_{k}^{-}\right\}$are non-decreasing.

(iv) For any fixed $\gamma<0$ the sequence $\left\{\lambda_{k}^{+}(\gamma)\right\}$ is non-decreasing and for any fixed $\gamma>0$ the sequence $\left\{\lambda_{k}^{-}(\gamma)\right\}$ is non-increasing. Furthermore, for $\gamma<0$ the cluster point of the set $\sigma_{\gamma}(a, b)$ can be only the endpoint $b$ of the gap $(a, b)$ and for $\gamma>0$ this cluster point can be only the endpoint a.

Proof. Consider the pencil of operators $\Phi(\gamma)$, defined by (3.3). By Proposition (3.1), the set $\sigma_{\gamma}(a, b)$ coincides with the spectrum $\sigma(\Phi(\gamma))$ of the pencil $\Phi(\gamma)$ and, furthermore,

$$
\operatorname{dim}\left(\operatorname{ker}\left(H_{\gamma}-\lambda I\right)\right)=\operatorname{dim}\left(\operatorname{ker}\left(I+\gamma X_{V}(\lambda)\right)\right) \forall \lambda \in \sigma_{\gamma}(a, b)
$$

Let $\mu_{k}^{+}(\lambda), \mu_{k}^{-}(\lambda)$ be positive and negative characteristic branches of the operator $H_{0}$ with respect to the operator $V$ on the gap (a,b) (see (3.6), (3.7) and Definition 3.2). 
The above arguments imply that the set $\sigma_{\gamma}(a, b)$ coincides with the union of the sets of solutions of the equations:

$$
\begin{aligned}
& \mu_{k}^{+}(\lambda)=-\frac{1}{\gamma}, \quad(k=1,2, \ldots) . \\
& \mu_{k}^{-}(\lambda)=-\frac{1}{\gamma}(k=1,2, \ldots) .
\end{aligned}
$$

Notice that, in view of (3.39), the following property is valid: if $\lambda(\gamma)$ is a solution of the equation (3.40), then the multiplicity of the eigenvalue $\lambda(\gamma)$ of the operator $H_{\gamma}$ coincides with the number of repetitions of the number $\mu_{k}^{+}(\lambda(\gamma))$ in the sequence (3.6) with $\lambda=\lambda(\gamma)$. The analogous property is valid for the equation (3.41). ${ }^{4}$ Observe that in the case $\gamma<0$ we need to consider the equation (3.40) only, because the equation (3.41) has no solution for any $k$. By a similar argument, in case $\gamma>0$ we need to consider only the equation (3.41).

Consider the case of a negative $\gamma$. By Proposition 3.7, all the characteristic branches $\mu_{k}^{+}(\lambda)$ increase on their domains $\left(\eta_{k}^{+}, b\right)$ and are continuous there. This fact implies that the equation (3.40) has a unique solution $\lambda_{k}^{+}(\gamma)$ if and only if $\gamma \in\left(\gamma_{k}^{+}, \tilde{\gamma}_{k}^{+}\right)$, where

$$
\begin{gathered}
\tilde{\gamma}_{k}^{+}=-\frac{1}{\lim _{\lambda \uparrow b} \mu_{k}^{+}(\lambda)}, \\
\gamma_{k}^{+}=-\frac{1}{\lim _{\lambda \downarrow \eta_{k}^{+}} \mu_{k}^{+}(\lambda)} .
\end{gathered}
$$

This means that for $\gamma<0$ representation (3.30) is valid, in which the domain $\operatorname{Dom}\left(\lambda_{k}^{+}\right)$ has the form (3.33). Furthermore, it is clear that $\lambda_{k}^{+}(\gamma)$ is continuous and increasing on this domain. Relations (3.34) and (3.35) are obvious. Property (3.36) follows from property (3.24) of characteristic branches (Proposition 3.7). Property (3.37) follows from the fact: if $\eta_{k}^{+}=-\infty$, then, in view of estimate

$$
\left\|X_{V}(\lambda)\right\| \leq\left\|V^{\frac{1}{2}}\right\|^{2}\left\|R_{\lambda}\left(H_{0}\right)\right\| \leq \frac{\|V\|}{\operatorname{dist}\left(\lambda, \sigma\left(H_{0}\right)\right)}=\frac{\|V\|}{b-\lambda}(\lambda<b),
$$

the relation holds

$$
\lim _{\lambda \downarrow-\infty} \mu_{k}^{+}(\lambda)=0 .
$$

The non-increase of the sequence $\left\{\mu_{k}^{+}(\lambda)\right\}$ for any fixed $\lambda \in(a, b)$ implies the nondecrease of the sequence $\left\{\lambda_{k}^{+}(\gamma)\right\}$ for any fixed $\gamma<0$ and the non-increase of the sequences $\left\{\gamma_{k}^{+}\right\},\left\{\tilde{\gamma}_{k}^{+}\right\}$defined by (3.42), (3.43). In view of Proposition 3.1, the sequence $\left\{\lambda_{k}^{+}(\gamma)\right\}$ can cluster only to the endpoint $b$.

The case $\gamma>0$ is treated analogously.

Remark 3.12. Proposition 3.11 says that, while $\gamma$ varies from 0 to $-\infty$, each of the eigenvalues $\lambda_{k}^{+}(\gamma)$ of the operator $H_{\gamma}$ enters the gap $(a, b)$ across the endpoint $b$ at the moment $\gamma=\tilde{\gamma}_{k}^{+}$and afterwards it either stays in $(a, b)$ for any $\gamma<0$ and tends to a point $\eta_{k}^{+} \in(a, b)$ as $\gamma \downarrow-\infty$, or (if $a=-\infty$ ) it tends to $-\infty$ as $\gamma \downarrow-\infty$, or (if $a>-\infty)$ it leaves the gap $(a, b)$ across the endpoint $a$ at the moment $\gamma=\gamma_{k}^{+}>-\infty$.

\footnotetext{
${ }^{4}$ It is clear that $\lambda(\gamma)$ cannot be a solution of the equations (3.40) and (3.41) simultaneously.
} 
In the analogous manner, while $\gamma$ varies from 0 to $+\infty$, each of the eigenvalues $\lambda_{k}^{-}(\gamma)$ of the operator $H_{\gamma}$ enters the gap $(a, b)$ across the endpoint $a$ at the moment $\gamma=\tilde{\gamma}_{k}^{-}$ and afterwards it either stays in $(a, b)$ for any $\gamma>0$ and tends to a point $\eta_{k}^{-} \in(a, b)$ as $\gamma \uparrow+\infty$, or (if $b=+\infty$ ) it tends to $+\infty$ as $\gamma \downarrow+\infty$, or (if $b<+\infty$ ) it leaves the gap $(a, b)$ across the endpoint $b$ at the moment $\gamma=\gamma_{k}^{-}<+\infty$.

Let us introduce the following

Definition 3.13. We call the function $\lambda_{k}^{+}(\gamma)$, defined by (3.31), the $k$-th branch of eigenvalues of the operator $H_{\gamma}$ which enter the gap $(a, b)$ of $\sigma\left(H_{0}\right)$ across the endpoint $b$ at the moment $\gamma=\tilde{\gamma}_{k}^{+}$in the sense that (3.34) is valid. In the analogous manner, we call the function $\lambda_{k}^{-}(\gamma)$, defined by (3.32), the $k$-th branch of eigenvalues of the operator $H_{\gamma}$ which enter the gap $(a, b)$ of $\sigma\left(H_{0}\right)$ across the endpoint a at the moment $\gamma=\tilde{\gamma}_{k}^{-}$in the sense that (3.38) is valid.

Definition 3.14. Let $\lambda_{k}^{+}(\gamma)$ be the $k$-th branch of eigenvalues of the operator $H_{\gamma}$ which enters the gap $(a, b)$ of $\sigma\left(H_{0}\right)$ across the endpoint $b$ at the moment $\gamma=0$. We call it a branch of virtual eigenvalues of the operator $H_{\gamma}$ at the endpoint $b$ of $(a, b)$. This means that

$$
\lim _{\gamma \uparrow 0} \lambda_{k}^{+}(\gamma)=b
$$

In the analogous manner we define a branch of virtual eigenvalues $\lambda_{k}^{-}(\gamma)$ of the operator $H_{\gamma}$ at the endpoint a of $(a, b)$. This branch satisfies the condition:

$$
\lim _{\gamma \downarrow 0} \lambda_{k}^{-}(\gamma)=a
$$

Remark 3.15. If $a=-\infty$ and $\gamma<0$, the branches of eigenvalues $\lambda_{k}^{+}(\gamma)$ of the operator $H_{\gamma}$ can be defined by the following ordering of the set $\sigma_{\gamma}(a, b)$ defined by $(3.2)$ :

$$
\lambda_{1}^{+}(\gamma) \leq \lambda_{2}^{+}(\gamma) \leq \cdots \leq \lambda_{k}^{+}(\gamma) \leq \cdots
$$

It is clear that all the branches of virtual eigenvalues at the endpoint $b$ (if they exist) occur at the beginning of this sequence. If $b=+\infty$ and $\gamma>0$, the branches of eigenvalues $\lambda_{k}^{-}(\gamma)$ of the operator $H_{\gamma}$ can be defined analogously and the branches of virtual eigenvalues at the endpoint $a$ have the analogous property.

The following statement about the branches of virtual eigenvalues is valid:

Proposition 3.16. Assume that the endpoint b of the gap $(a, b)$ of $\sigma\left(H_{0}\right)$ is finite. If

$$
l(b)=M\left(b, H_{0}, V\right)<\infty,
$$

then the operator $H_{\gamma}$ has exactly $l(b)$ branches

$$
a<\lambda_{1}^{+}(\gamma) \leq \lambda_{2}^{+}(\gamma) \leq \cdots \leq \lambda_{l(b)}^{+}(\gamma)
$$

of virtual eigenvalues at the endpoint $b$ of $(a, b)$. Furthermore, for any $1 \leq k \leq l(b)$ and $\gamma \in \operatorname{Dom}\left(\lambda_{k}^{+}\right)$,

$$
\lambda_{k}^{+}(\gamma)=\left(\mu_{k}^{+}\right)^{-1}\left(-\frac{1}{\gamma}\right)
$$


where $\left(\mu_{k}^{+}\right)^{-1}$ is the inverse of the function $\mu_{k}^{+}(\lambda)$ (the main characteristic branch of $H_{0}$ with respect to $V$ near the endpoint b, see Definition 3.9). If (3.45) holds and $a=-\infty$, then

$$
\operatorname{Dom}\left(\lambda_{k}^{+}\right)=(-\infty, 0) \forall k \in\{1,2, \ldots, l(b)\} .
$$

Hence the operator $H_{\gamma}$ has at least $l(b)$ eigenvalues in the gap $(-\infty, b)$ for any $\gamma<0$. If

$$
M\left(b, H_{0}, V\right)=\infty,
$$

then the number of the branches of eigenvalues $\left\{\lambda_{k}^{+}(\gamma)\right.$ of the operator $H_{\gamma}$, which go into the gap $(a, b)$ across the endpoint $b$, is infinite, each of them is a virtual eigenvalue and the property is valid for them

$$
\left(-\theta_{k}, 0\right) \subseteq \operatorname{Dom}\left(\lambda_{k}^{+}\right), \text {where } \theta_{k} \uparrow+\infty \text { for } k \rightarrow \infty .
$$

The latter fact means that the operator $H_{\gamma}$ has the infinite number of eigenvalues in the gap $(a, b)$ for any $\gamma<0$. These eigenvalues cluster to the endpoint $b$ only and formula (3.46) with $l(b)=\infty$ is valid for them.

For the branches of virtual eigenvalues of the operator $H_{\gamma}$ at the endpoint a of $(a, b)$ the analogous assertions are valid as in the case of the endpoint $b$.

Proof. Assume that (3.45) is valid. By Definition 3.9 of the asymptotic multiplicity $l(b)$ and the main characteristic branches $\left\{\mu_{k}^{+}(\lambda)\right\}_{k=1}^{l(b)}$,

$$
\mu_{k, b}^{+}=\lim _{\lambda_{\uparrow} b} \mu_{k}^{+}(\lambda) \begin{cases}=+\infty, & \text { if } 1 \leq k \leq l(b), \\ <+\infty, & \text { if } k>l(b) .\end{cases}
$$

We see from this relation and (3.31) that

$$
\operatorname{Dom}\left(\lambda_{k}^{+}\right)=\left(\gamma_{k}^{+}, 0\right) \forall k \in\{1,2, \ldots, l(b)\}
$$

hence the functions $\left\{\lambda_{k}^{+}(\gamma)\right\}_{k=1}^{l(b)}$ are branches of virtual eigenvalues of the operator $H_{\gamma}$ at the endpoint $b$ of $(a, b)$. Taking into account the increase of the characteristic branches, we see from (3.50) that for $\gamma \in\left(-\frac{1}{\mu_{l(b)+1, b}^{+}}, 0\right)$ and $k>l(b)$ the equation (3.40) has no solution in the gap $(a, b)$. This means that for $k>l(b)$ the equation (3.40) gives no virtual branch of eigenvalues of $H_{\gamma}$ at the endpoint $b$ of $(a, b)$. So, we have proved that all the branches of virtual eigenvalues of $H_{\gamma}$ at the endpoint $b$ of $(a, b)$ are exhausted by the sequence $\left\{\lambda_{k}^{+}(\gamma)\right\}_{k=1}^{l(b)}$, and, in view of $(3.31)$, they are expressed by formula (3.46).

Assume that $a=-\infty$ and that, as above, (3.45) is valid. In view of (3.36), (3.37) and (3.51), for the branches of virtual eigenvalues of $H_{\gamma}$ at the endpoint $b$ the equalities (3.47) are valid.

We now turn to the case (3.48). Assume that $a>-\infty$. By Proposition 3.10, all the positive characteristic branches are the main characteristic branches near the endpoint $b$, i,e., they have the property:

$$
\lim _{\lambda \uparrow b} \mu_{k}^{+}(\lambda)=+\infty \quad \forall k \in \mathbb{N}
$$

As in the case of a finite $l(b)$, this property implies that all the functions $\lambda_{k}^{+}(\gamma) \quad(k \in$ $\mathbb{N})$, defined by (3.31), are branches of virtual eigenvalues of $H_{\gamma}$ at the endpoint $b$ 
of $(a, b)$. Let us prove property (3.49). By Proposition 3.7, the sequence $\left\{\eta_{k}^{+}\right\}_{k \in \mathbb{N}}$, defined by (3.22)), is non-decreasing. Consider two cases:

$$
\lim _{k \rightarrow \infty} \eta_{k}^{+}=b
$$

and

$$
\eta_{\infty}^{+}=\lim _{k \rightarrow \infty} \eta_{k}^{+}<b
$$

In the case (3.53) there exists $k_{0} \in \mathbb{N}$, such that for any $k \geq k_{0} \eta_{k}^{+} \in(a, b)$, hence, in view of $(3.44)$ and $(3.52), \operatorname{Im}\left(\mu_{k}^{+}\right)=(0, \infty)$ for these $k$. The latter fact implies that

$$
\operatorname{Dom}\left(\lambda_{k}^{+}\right)=(-\infty, 0) \text { for } k \geq k_{0}
$$

hence property (3.49) is valid. In case (3.54) holds a point $\lambda_{0} \in\left(\eta_{\infty}^{+}, b\right)$ belongs to $\operatorname{Dom}\left(\mu_{k}^{+}\right)$for any $k \in \mathbb{N}$ and the numbers $\mu_{k}^{+}\left(\lambda_{0}\right)$ tend to zero for $k \rightarrow \infty$, because they are the eigenvalues of the compact operator $X_{V}\left(\lambda_{0}\right)$. Since, in view of (3.52),

$$
\left(\mu_{k}^{+}\left(\lambda_{0}\right),+\infty\right) \subseteq \operatorname{Im}\left(\mu_{k}^{+}\right),
$$

then property (3.49) is valid. This property and Proposition 3.11 imply that for any $\gamma<0$ the set $\sigma_{\gamma}(a, b)$, defined by (3.2), consists of infinite sequence of the form $\left\{\lambda_{k}^{+}(\gamma)\right\}_{k \geq k_{0}(\gamma)}$ whose terms are expressed by formula (3.46), and this sequence can cluster to the endpoint $b$ only. The case $a=-\infty$ is treated in the same manner as in the case of a finite $l(b)$.

The following proposition deals with estimates from below of the asymptotic multiplicity and the main characteristic branches.

Proposition 3.17. Assume that there exists a subspace $L_{n} \subset \mathcal{H}$ of a finite dimension $n$ and an increasing function $\mu(\lambda)$ defined on $\left.\left(\lambda_{0}, b\right)\left(\lambda_{0} \in(a, b)\right)\right)$ such that

$$
\begin{gathered}
\mu(\lambda)>0 \quad \forall \lambda \in\left(\lambda_{0}, b\right), \\
\mu(\lambda) \uparrow \infty \text { as } \lambda \uparrow b,
\end{gathered}
$$

and

$$
\inf _{x \in L_{n},\|x\|=1}\left(X_{V}(\lambda) x, x\right) \geq \mu(\lambda),
$$

where $X_{V}(\lambda)$ is defined by (3.4). Then

$$
M\left(b, H_{0}, V\right) \geq n,
$$

and for the first $n$ main characteristic branches $\left\{\mu_{k}^{+}(\lambda)\right\}_{k=1}^{n}$ of $H_{0}$ (with respect to $V$ near b) the property

$$
\left(\lambda_{0}, b\right) \subseteq \operatorname{Dom}\left(\mu_{k}^{+}\right) \forall k \in\{1,2, \ldots, n\}
$$

and the estimates

$$
\mu_{k}^{+}(\lambda) \geq \mu(\lambda) \forall \lambda \in\left(\lambda_{0}, 0\right), k \in\{1,2, \ldots, n\}
$$

are valid. 
Proof. In order to prove property (3.59), we need to show that for any $\lambda \in\left(\lambda_{0}, b\right)$ there exist at least $n$ positive eigenvalues of the operator $X_{V}(\lambda)$. Indeed, otherwise the invariant subspace $G_{\lambda}^{-} \subseteq \mathcal{H}$ of the operator $X_{V}(\lambda)$, corresponding to its non-positive eigenvalues, has codimension less than $n$. Then there exist a vector $g \neq 0$ belonging to $L_{n} \cap G^{-}(\lambda)$. Hence, in view of (3.55) and (3.57), $\left(X_{V}(\lambda) g, g\right)>0$. On the other hand, by the choice of the subspace $G^{-}(\lambda),\left(X_{V}(\lambda) g, g\right) \leq 0$. This contradiction proves property (3.59).

Let us prove property (3.60). Take $\lambda \in\left(\lambda_{0}, b\right)$ and consider the invariant subspace $E_{n-1}(\lambda)$ of the operator $X_{V}(\lambda)$ corresponding to the first $n-1$ positive eigenvalues of it $\left\{\mu_{k}^{+}(\lambda)\right\}_{k=1}^{n-1}$. Denote $G(\lambda)=\left(E_{n-1}(\lambda)\right)^{\perp}$. Since $\operatorname{codim}(G(\lambda))=n-1$, there exists a vector $e \in L_{n} \cap G(\lambda),\|e\|=1$. Then, if $\mu_{n}^{+}(\lambda)$ is the $n$-th positive eigenvalue of $X_{V}(\lambda)$, we have, taking into account condition (3.57),

$$
\mu_{n}^{+}(\lambda)=\max _{x \in G,\|x\|=1}\left(X_{V}(\lambda) x, x\right) \geq\left(X_{V}(\lambda) e, e\right) \geq \inf _{x \in L_{n},\|x\|=1}\left(X_{V}(\lambda) x, x\right) \geq \mu(\lambda) .
$$

This estimate and condition (3.56) imply that $\left\{\mu_{k}^{+}(\lambda)\right\}_{k=1}^{n}$ are the main characteristic branches of $H_{0}$ near the endpoint $b$ of the gap $(a, b)$, hence estimate (3.58) is valid. Moreover, we have proved estimate (3.60).

Propositions 3.17, 3.11 and 3.16 imply

Corollary 3.18. Assume that all the conditions of Proposition 3.17 are satisfied. Then the first $n$ branches of eigenvalues of the operator $H_{\gamma}$, gone into the gap $(a, b)$ of $\sigma\left(H_{0}\right)$ across the endpoint $b$, are virtual and the following estimate is valid for them for a small enough $\gamma<0$ :

$$
\lambda_{k}^{+}(\gamma) \leq \mu^{-1}\left(-\frac{1}{\gamma}\right) \quad \forall k \in\{1,2, \ldots, n\},
$$

where $\mu^{-1}$ is the inverse of the function $\mu(\lambda)$ used in condition (3.57).

\section{Representations for the Birman-Schwinger operator of the Schrödinger operator}

4.1 ${ }^{\mathbf{0}}$. We return to the Schrödinger operator $H_{\gamma}$, defined by (1.1). Recall that the domain $\operatorname{Dom}\left(H_{\gamma}\right)$ of the operator $H_{\gamma}$ is described in the following manner:

$$
\operatorname{Dom}\left(H_{\gamma}\right)=\left\{u \in L_{2}\left(\mathbb{R}^{d}\right) \mid u \in W_{2, l o c}^{2 l}\left(\mathbb{R}^{d}\right), H_{\gamma} u \in L_{2}\left(\mathbb{R}^{d}\right)\right\} .
$$

In the sequel we shall assume that the potential $V(x)$ satisfies the conditions:

$$
\begin{gathered}
V(\cdot) \in C\left(\mathbb{R}^{d}\right), \\
V(\mathbf{x}) \geq 0 \quad \forall \mathbf{x} \in \mathbb{R}^{d}, \\
\lim _{|\mathbf{x}| \rightarrow \infty} V(\mathbf{x})=0 .
\end{gathered}
$$

Notice that the "unperturbed" operator $H_{0}=(-\Delta)^{l}$ is self-adjoint, because the Fourier transform on $\mathbb{R}^{d}$ establishes a unitary equivalence between it and the multiplication operator by the function $|\mathbf{p}|^{2 l}$ (where "p" denotes the variable in $\mathbb{R}^{d}$ ). In 
view of conditions (4.1) and (4.3), the operator $V(\mathbf{x})$. is bounded in $L_{2}\left(\mathbb{R}^{d}\right)$. Furthermore, it is self-adjoint, because the potential $V(\mathbf{x})$ is real-valued. These circumstances imply that the Schrödinger operator $H_{\gamma}$, defined by (1.1), is self-adjoint. Observe that the spectrum $\sigma\left(H_{0}\right)$ of the unperturbed operator $H_{0}$ coincides with the image of the function $|\mathbf{p}|^{2 l}$, that is $\sigma\left(H_{0}\right)=[0, \infty)$. Hence the spectrum $\sigma\left(H_{0}\right)$ has the unique gap $(-\infty, 0)$. We shall study the eigenvalues of the operator $H_{\gamma}$ in this gap. In order to use the general theory (developed in Section 3), we need to show that, under conditions (4.1)-(4.3) imposed on the potential $V(\mathbf{x})$, condition (B) (formulated in the beginning of Section 3 ) holds. Recall that we denote briefly the operator $V(\mathbf{x}) \cdot$ by $V$. Observe that, in view of condition (4.2), $V \geq 0$.

In what follows we shall need the following well known statement (see [Gl]):

Proposition 4.1. For $\lambda_{0}<0$ the operator $V^{\frac{1}{2}} R_{\lambda_{0}}\left(H_{0}\right)$ is compact.

¿From the above proposition and the fact that the operator $V$ is bounded we obtain the following

Corollary 4.2. Under conditions (4.1), (4.2), (4.3) the operators $H_{0}, V$ satisfy condition (B) of Section 3.

In the next two subsections of this section we deal with an extraction of a finiterank portion from the Birman-Schwinger operator $X_{V}(\lambda)$ of the operator $H_{\gamma}$.

4.2 ${ }^{\circ}$. Consider the case of $\mathbb{R}^{d}$ with $d$ odd. We shall need the following result.

Lemma 4.3. If $d$ is odd, $2 l>d$ and $\lambda<0$, then the resolvent $R_{\lambda}\left(H_{0}\right)$ of the operator

$$
H_{0}=(-\Delta)^{l}
$$

is the convolution operator

$$
R_{\lambda}\left(H_{0}\right) f=K \star f
$$

where $K=K(\mathbf{x}, \lambda)$ is a continuous function belonging to $L_{2}\left(\mathbb{R}^{d}\right)$ and having the following representation:

$$
K(\mathbf{x}-\mathbf{y}, \lambda)=F(\mathbf{x}, \mathbf{y}, \lambda)+\Theta(\mathbf{x}-\mathbf{y}, \lambda),
$$

where

$$
\begin{gathered}
F(\mathbf{x}, \mathbf{y}, \lambda)=(2 \pi)^{\frac{d}{2}} \sum_{|\mathbf{k}+\mathbf{n}| \leq 2 l-d-1}|\lambda|^{\frac{|\mathbf{k}+\mathbf{n}|+d}{2 l}-1} \frac{(i \mathbf{x})^{\mathbf{k}}}{\mathbf{k} !} \frac{(-i \mathbf{y})^{\mathbf{n}}}{\mathbf{n} !} \xi_{\mathbf{k}+\mathbf{n}}, \\
\xi_{\mathbf{k}}=(2 \pi)^{-d} \int_{\mathbb{R}^{d}} \frac{\mathbf{s}^{\mathbf{k}} d \mathbf{s}}{|\mathbf{s}|^{2 l}+1}
\end{gathered}
$$

and for the function $\Theta(\mathbf{x}, \lambda)$ the estimate

$$
|\Theta(\mathbf{x}, \lambda)| \leq M|\mathbf{x}|^{2 l-d}(\lambda<0),
$$

is valid, in which $M>0$ does not depend on $\mathbf{x}, \lambda$. 
Proof. Since the Fourier transform on $\mathbb{R}^{d}$ establishes a unitary equivalence between the operator $H_{0}$, defined by (4.4), and the multiplication operator $\hat{H}_{0}=|\mathbf{p}|^{2 l}$, the resolvent $R_{\lambda}\left(H_{0}\right)$ is the convolution operator (4.5), in which

$$
K=K(\mathbf{x}, \lambda)=(2 \pi)^{-\frac{d}{2}} \int_{\mathbb{R}^{d}} \frac{\exp (i \mathbf{p} \cdot \mathbf{x}) d \mathbf{p}}{|\mathbf{p}|^{2 l}+|\lambda|} .
$$

Since $\frac{1}{|\mathbf{p}|^{2 l}+|\lambda|} \in L_{2}\left(\mathbb{R}^{d}\right) \cap L_{1}\left(\mathbb{R}^{d}\right)$, the function $K(\mathbf{x}, \lambda)$ is continuous and belongs to $L_{2}\left(\mathbb{R}^{d}\right)$. Using the Taylor expansion of the exponent, we obtain:

$$
K(\mathbf{x}, \lambda)=\tilde{F}(\mathbf{x}, \lambda)+\Theta(\mathbf{x}, \lambda),
$$

where

$$
\begin{gathered}
\tilde{F}(\mathbf{x}, \lambda)=(2 \pi)^{-\frac{d}{2}} \sum_{k=0}^{2 l-d-1} \frac{1}{k !} \int_{\mathbb{R}^{d}} \frac{(i \mathbf{p} \cdot \mathbf{x})^{k} d \mathbf{p}}{|\mathbf{p}|^{2 l}+|\lambda|}, \\
\Theta(\mathbf{x}, \lambda)=(2 \pi)^{-\frac{d}{2}} \int_{\mathbb{R}^{d}} \frac{\left(\exp (i \mathbf{p} \cdot \mathbf{x})-\sum_{k=0}^{2 l-d-1} \frac{(i \mathbf{p} \cdot \mathbf{x})^{k}}{k !}\right) d \mathbf{p}}{|\mathbf{p}|^{2 l}+|\lambda|} .
\end{gathered}
$$

It is not difficult to see that the integrals in (4.11), (4.12) converge absolutely. Furthermore,

$$
\Theta(\mathbf{x}, \lambda)=(2 \pi)^{-\frac{d}{2}} \int_{\mathbb{R}^{d}} \frac{\left(\cos (\mathbf{p} \cdot \mathbf{x})-\sum_{\nu=0}^{l-\frac{d+1}{2}}(-1)^{\nu} \frac{(\mathbf{p} \cdot \mathbf{x})^{2 \nu}}{(2 \nu) !}\right) d \mathbf{p}}{|\mathbf{p}|^{2 l}+|\lambda|},
$$

because in (4.12) the integral of the odd part of the integrand vanishes. Changing the variables in the latter integral $\mathbf{s}=|\mathbf{x}| \mathbf{p}$ and rotating the space $\mathbb{R}^{d}$ such that the direction of the vector $\mathbf{x}$ transforms to the direction of the axis $s_{1}$, we obtain the estimate $|\Theta(\mathbf{x}, \lambda)| \leq M|\mathbf{x}|^{2 l-d}$, where

$$
M=(2 \pi)^{-\frac{d}{2}} \int_{\mathbb{R}^{d}} \frac{\left|\cos s_{1}-\sum_{\nu=0}^{l-\frac{d+1}{2}}(-1)^{\nu} \frac{s_{1}^{2 \nu}}{(2 \nu) !}\right| d \mathbf{s}}{|\mathbf{s}|^{2 l}} .
$$

Observe that the latter integral converges, because

$$
\left|\cos s-\sum_{\nu=0}^{l-\frac{d+1}{2}}(-1)^{\nu} \frac{s^{2 \nu}}{(2 \nu) !}\right| \leq \begin{cases}C_{1}|s|^{2 l-d+1} & \text { for }|s| \leq 1, \\ C_{2}|s|^{2 l-d-1} & \text { for }|s| \geq 1,\end{cases}
$$

where $C_{1}, C_{2}>0$ do not depend on $s$. Thus the function $\Theta(\mathbf{x}, \lambda)$ satisfies estimate (4.8) in which $M$ is expressed by (4.13).

In view of (4.10), in order to prove representation (4.6), it remains only to show that

$$
\tilde{F}(\mathbf{x}-\mathbf{y}, \lambda)=F(\mathbf{x}, \mathbf{y}, \lambda) .
$$

One has:

$$
\begin{array}{r}
\int_{\mathbb{R}^{d}} \frac{(i \mathbf{p} \cdot(\mathbf{x}-\mathbf{y}))^{k} d \mathbf{p}}{|\mathbf{p}|^{2 l}+|\lambda|}=|\lambda|^{\frac{k+d}{2 l}-1} \int_{\mathbb{R}^{d}} \frac{(i \mathbf{s} \cdot(\mathbf{x}-\mathbf{y}))^{k} d \mathbf{s}}{|\mathbf{s}|^{2 l}+1}= \\
=|\lambda|^{\frac{k+d}{2 l}-1} \int_{\mathbb{R}^{d}} \frac{\left(\sum_{\nu=1}^{d} s_{\nu}\left(i x_{\nu}-i y_{\nu}\right)\right)^{k} d \mathbf{s}}{|\mathbf{s}|^{2 l}+1}= \\
=k !|\lambda|^{\frac{k+d}{2 l}-1} \sum_{|\mathbf{k}+\mathbf{n}|=k} \frac{(i \mathbf{x})^{\mathbf{k}}}{\mathbf{k} !} \frac{(-i \mathbf{y})^{\mathbf{n}}}{\mathbf{n} !} \int_{\mathbb{R}^{d}} \frac{\mathbf{s}^{\mathbf{k}+\mathbf{n}} d \mathbf{s}}{|\mathbf{s}|^{2 l}+1} .
\end{array}
$$


The latter equality and (4.11), (4.7) imply (4.14). The lemma is proven.

We now turn to the main statement of this subsection.

Proposition 4.4. Assume that $d$ is odd, $2 l>d$ and that the potential $V(\mathbf{x})$ satisfies the additional condition:

$$
\int_{\mathbb{R}^{d}}|\mathbf{x}|^{2(2 l-d)} V(\mathbf{x}) d \mathbf{x}<\infty .
$$

Then the Birman-Schwinger operator

$$
X_{V}(\lambda)=V^{\frac{1}{2}} R_{\lambda}\left(H_{0}\right) V^{\frac{1}{2}}
$$

of the operator $H_{\gamma}$ has the representation for $\lambda<0$ :

$$
X_{V}(\lambda)=\Phi(\lambda)+T(\lambda),
$$

where $\Phi(\lambda)$ is an integral operator with the kernel

$$
\Phi(\mathbf{x}, \mathbf{y}, \lambda)=(2 \pi)^{-\frac{d}{2}}(V(\mathbf{x}))^{\frac{1}{2}} F(\mathbf{x}, \mathbf{y}, \lambda)(V(\mathbf{y}))^{\frac{1}{2}},
$$

$F(\mathbf{x}, \mathbf{y}, \lambda)$ is defined by (4.7), $T(\lambda)$ is an operator belonging to the Hilbert-Schmidt class $\mathcal{S}_{2}$ and its Hilbert-Schmidt norm is bounded uniformly with respect to $\lambda<0$, that is

$$
\exists \bar{T}>0 \forall \lambda<0: \quad\|T(\lambda)\|_{2} \leq \bar{T} .
$$

Proof. Let $\Theta(\mathbf{x}, \lambda)$ be the function used in representation (4.6) of the kernel $K(\mathbf{x}-$ $\mathbf{y}, \lambda)$ of the integral operator $(2 \pi)^{\frac{d}{2}} R_{\lambda}\left(H_{0}\right)$. Then, in order to prove the proposition, we should show that for any $\lambda<0$ the integral operator $T(\lambda)$ with the kernel

$$
T(\mathbf{x}, \mathbf{y}, \lambda)=(2 \pi)^{-\frac{d}{2}}(V(\mathbf{x}))^{\frac{1}{2}} \Theta(\mathbf{x}-\mathbf{y}, \lambda)(V(\mathbf{y}))^{\frac{1}{2}}
$$

has the desired property. In view of estimate (4.8), it is enough to show that the kernel $(V(\mathbf{x}))^{\frac{1}{2}}|\mathbf{x}-\mathbf{y}|^{2 l-d}(V(\mathbf{y}))^{\frac{1}{2}}$ belongs to the Hilbert-Schmidt class. We have:

$$
\int_{\mathbb{R}^{d}} V(\mathbf{x}) d \mathbf{x} \int_{\mathbb{R}^{d}}|\mathbf{x}-\mathbf{y}|^{2(2 l-d)} V(\mathbf{y}) d \mathbf{y} \leq \int_{\mathbb{R}^{d}} V(\mathbf{x}) d \mathbf{x} \int_{\mathbb{R}^{d}}(|\mathbf{x}|+|\mathbf{y}|)^{2(2 l-d)} V(\mathbf{y}) d \mathbf{y}
$$

By virtue of condition (4.16), the latter integral converges.

It turns out that the numbers $\xi_{\mathbf{k}}$, used in formula (4.7), can be calculated by a standard method. Let us formulate the corresponding statement.

Proposition 4.5. Let $\mathbf{k} \in \mathbb{Z}_{+}^{d}, l>0$ be such that $|\mathbf{k}|<2 l-d$. Consider the integral

$$
\xi_{\mathbf{k}}=(2 \pi)^{-d} \int_{\mathbb{R}^{d}} \frac{\mathbf{s}^{\mathbf{k}} d \mathbf{s}}{|\mathbf{s}|^{2 l}+1}(|\mathbf{k}|<2 l-d) .
$$

Then:

(i) For any permutation $\Pi \mathbf{k}$ of components of the multi-index $\mathbf{k}: \quad \xi_{\Pi \mathbf{k}}=\xi_{\mathbf{k}}$;

(ii) If at least one component of the multi-index $\mathbf{k}$ is odd then $\xi_{\mathbf{k}}=0$; 
(iii) If all the components of the multi-index $\mathbf{k}$ are even, we write $\mathbf{k}=2 \mathbf{m}$ with $\mathbf{m}=\left(m_{1}, m_{2}, \ldots, m_{d}\right) \in \mathbb{Z}_{+}^{d}$, and then

$$
\xi_{\mathbf{k}}=(2 \pi)^{-d} \frac{\pi}{l \sin \left(\frac{\pi}{l}\left(\frac{d}{2}+|\mathbf{m}|\right)\right)} \frac{\prod_{j=1}^{d} \Gamma\left(m_{j}+\frac{1}{2}\right)}{\Gamma\left(\frac{d}{2}+|\mathbf{m}|\right)} .
$$

4.3. $\mathbf{3}^{\mathbf{0}}$. We now turn to the case of $\mathbb{R}^{d}$ with $d$ even. The following lemma plays the same role as Lemma 4.3 in the case of an odd dimension:

Lemma 4.6. If $d$ is even, $2 l \geq d$ and $\lambda<0$, then the resolvent $R_{\lambda}\left(H_{0}\right)$ of the operator $H_{0}$, defined by (4.4), is the convolution operator (4.5), in which the function $K=K(\mathbf{x}, \lambda)$ belongs to $L_{2}\left(\mathbb{R}^{d}\right)$, and for $2 l>d$ it has the following representation:

$$
K(\mathbf{x}-\mathbf{y}, \lambda)=F(\mathbf{x}, \mathbf{y}, \lambda)+\Theta(\mathbf{x}-\mathbf{y}, \lambda),
$$

where

$$
\begin{aligned}
F(\mathbf{x}, \mathbf{y}, \lambda)=2 l(2 \pi)^{\frac{d}{2}} \sum_{|\mathbf{k}+\mathbf{n}| \leq 2 l-d-1} \frac{|\lambda| \frac{|\mathbf{k}+\mathbf{n}|+d}{2 l}-1}{2 l-d-|\mathbf{k}+\mathbf{n}|} \frac{(i \mathbf{x})^{\mathbf{k}}}{\mathbf{k} !} \frac{(-i \mathbf{y})^{\mathbf{n}}}{\mathbf{n} !} \eta_{\mathbf{k}+\mathbf{n}}+ \\
+(2 \pi)^{\frac{d}{2}} \ln \left(\frac{1}{|\lambda|}\right)_{|\mathbf{k}+\mathbf{n}|=2 l-d} \frac{(i \mathbf{x})^{\mathbf{k}}}{\mathbf{k} !} \frac{(-i \mathbf{y})^{\mathbf{n}}}{\mathbf{n} !} \eta_{\mathbf{k}+\mathbf{n}}
\end{aligned}
$$

or in another form:

$$
\begin{aligned}
& F(\mathbf{x}, \mathbf{y}, \lambda)=(2 \pi)^{\frac{d}{2}} \sum_{|\mathbf{k}+\mathbf{n}| \leq 2 l-d-1}|\lambda|^{\frac{|\mathbf{k}+\mathbf{n}|+d}{2 l}-1} \frac{(i \mathbf{x})^{\mathbf{k}}}{\mathbf{k} !} \frac{(-i \mathbf{y})^{\mathbf{n}}}{\mathbf{n} !} \xi_{\mathbf{k}+\mathbf{n}}+ \\
&+(2 \pi)^{\frac{d}{2}} \ln \left(\frac{1}{|\lambda|}\right) \sum_{|\mathbf{k}+\mathbf{n}|=2 l-d} \frac{(i \mathbf{x})^{\mathbf{k}}}{\mathbf{k} !} \frac{(-i \mathbf{y})^{\mathbf{n}}}{\mathbf{n} !} \eta_{\mathbf{k}+\mathbf{n}}
\end{aligned}
$$

where

$$
\eta_{\mathbf{k}}=(2 \pi)^{-d} \int_{\mathbb{R}^{d}} \frac{\mathbf{s}^{\mathbf{k}} d \mathbf{s}}{\left(|\mathbf{s}|^{2 l}+1\right)^{2}}
$$

and the numbers $\xi_{\mathbf{k}}$ are defined by (4.19). Furthermore, for the function $\Theta(\mathbf{x}, \lambda)$ the estimate

$$
|\Theta(\mathbf{x}, \lambda)| \leq M\left(1+|\mathbf{x}|^{2 l-d}\right)(1+\ln (|\mathbf{x}|+1))
$$

holds for a small enough $\lambda<0$, in which $M>0$ does not depend on $\mathbf{x}, \lambda$.

If $2 l=d$, then the representation

$$
K(\mathbf{x}-\mathbf{y}, \lambda)=F_{1}(\lambda)+\Theta_{1}(\mathbf{x}-\mathbf{y})+\Theta_{2}(\mathbf{x}-\mathbf{y}, \lambda)
$$

is valid, where

$$
F_{1}(\lambda)=(2 \pi)^{\frac{d}{2}} \ln \left(\frac{1}{|\lambda|}\right) \eta_{\mathrm{o}}
$$

the function $\Theta_{1}(x)$ belongs to $L_{2}\left(\mathbb{R}^{d}\right)$ and for the function $\Theta_{2}(\mathbf{x}, \lambda)$ the estimate

$$
\left|\Theta_{2}(\mathbf{x}, \lambda)\right| \leq \tilde{M}\left(1+|\mathbf{x}|^{2 l-d}\right)(1+\ln (|\mathbf{x}|+1))
$$

holds for a small enough $\lambda<0$, in which $\tilde{M}>0$ does not depend on $\mathbf{x}, \lambda$. 
Proof. Like in the proof of Lemma 4.3, we show that the resolvent $R_{\lambda}\left(H_{0}\right)$ is the convolution operator (4.5) in which $K \in L_{2}\left(\mathbb{R}^{d}\right)$. But the further arguments of the proof of Lemma 4.3 fail, because for $|\mathbf{k}+\mathbf{n}|=2 l-d$ the integral $\int_{\mathbb{R}^{d}} \frac{\mathbf{s}^{\mathbf{k}+\mathbf{n}} d \mathbf{s}}{\mid \mathbf{s} \mathbf{s}^{2 l}+1}$ diverges. Therefore we are forced to use some trick in order to overcome this difficulty.

First assume that $2 l>d$. Denote $t=|\lambda|^{\frac{1}{2 t}}$. Then we have from (4.9):

$$
\begin{aligned}
K^{\prime}(\mathbf{x}, t)=\frac{\partial}{\partial t} K\left(\mathbf{x},-t^{2 l}\right) & =\frac{\partial}{\partial t}(2 \pi)^{-\frac{d}{2}} \int_{\mathbb{R}^{d}} \frac{\exp (i \mathbf{p} \cdot \mathbf{x}) d \mathbf{p}}{|\mathbf{p}|^{2 l}+t^{2 l}}= \\
= & -2 l t^{2 l-1}(2 \pi)^{-\frac{d}{2}} \int_{\mathbb{R}^{d}} \frac{\exp (i \mathbf{p} \cdot \mathbf{x}) d \mathbf{p}}{\left(|\mathbf{p}|^{2 l}+t^{2 l}\right)^{2}} .
\end{aligned}
$$

Observe that this differentiation is justified because the above integrals converge uniformly with respect to $t \in[\delta, \infty)$ for any $\delta>0$. We have from (4.27) using the Taylor expansion for the exponent and the change of variables $\mathbf{p}=t \mathbf{s}$ in the integral:

$$
\begin{aligned}
K^{\prime}(\mathbf{x}-\mathbf{y}, t) & =\frac{-2 l}{(2 \pi)^{\frac{d}{2}}} \sum_{k=0}^{2 l-d} \frac{1}{k !} \frac{1}{t^{2 l+1-d-k}} \int_{\mathbb{R}^{d}} \frac{(i \mathbf{s} \cdot(\mathbf{x}-\mathbf{y}))^{k} d \mathbf{s}}{\left(|\mathbf{s}|^{2 l}+1\right)^{2}}+\Theta^{\prime}(\mathbf{x}-\mathbf{y}, t)= \\
& =\frac{-2 l}{(2 \pi)^{\frac{d}{2}}} \sum_{k=0}^{2 l-d} \frac{1}{t^{2 l+1-d-k}} \sum_{|\mathbf{k}+\mathbf{n}|=k} \frac{(i \mathbf{x})^{\mathbf{k}}}{\mathbf{k} !} \frac{(-i \mathbf{y})^{\mathbf{n}}}{\mathbf{n} !} \int_{\mathbb{R}^{d}} \frac{\mathbf{s}^{\mathbf{k}+\mathbf{n}} d \mathbf{s}}{\left(|\mathbf{s}|^{2 l}+1\right)^{2}} \\
& +\Theta^{\prime}(\mathbf{x}-\mathbf{y}, t)
\end{aligned}
$$

where

$$
\begin{gathered}
\Theta^{\prime}(\mathbf{x})=-\frac{2 l}{t^{2 l-d+1}}\left(I(\mathbf{x}, t)+I_{\infty}(\mathbf{x}, t)\right), \\
I(\mathbf{x}, t)=\int_{|\mathbf{s}| \leq(|\mathbf{x}| t)^{-1}} \frac{C(t, \mathbf{s}, \mathbf{x}) d \mathbf{s}}{\left(|\mathbf{s}|^{2 l}+1\right)^{2}} \\
I_{\infty}(\mathbf{x}, t)=\int_{|\mathbf{s}| \geq(|\mathbf{x}| t)^{-1}} \frac{C(t, \mathbf{s}, \mathbf{x}) d \mathbf{s}}{\left(|\mathbf{s}|^{2 l}+1\right)^{2}}
\end{gathered}
$$

and

$$
C(t, \mathbf{s}, \mathbf{x})=\cos (t \mathbf{s} \cdot \mathbf{x})-\sum_{\nu=0}^{l-\frac{d}{2}}(-1)^{\nu} \frac{(t \mathbf{s} \cdot \mathbf{x})^{2 \nu}}{(2 \nu) !} .
$$

Taking into account (4.27) and (4.15), we can write $K^{\prime}(\mathbf{x}-\mathbf{y}, t)$ in another form:

$$
\begin{aligned}
K^{\prime}(\mathbf{x}-\mathbf{y}, t) & =(2 \pi)^{-\frac{d}{2}}\left\{\frac{\partial}{\partial t} \sum_{|\mathbf{k}+\mathbf{n}| \leq 2 l-d-1} t^{|\mathbf{k}+\mathbf{n}|-2 l+d} \frac{(i \mathbf{x})^{\mathbf{k}}}{\mathbf{k} !} \frac{(-i \mathbf{y})^{\mathbf{n}}}{\mathbf{n} !}\right. \\
& \left.\times \int_{\mathbb{R}^{d}} \frac{\mathbf{s}^{\mathbf{k}+\mathbf{n}} d \mathbf{s}}{|\mathbf{s}|^{2 l}+1}-\frac{2 l}{t} \sum_{|\mathbf{k}+\mathbf{n}|=2 l-d} \frac{(i \mathbf{x})^{\mathbf{k}}}{\mathbf{k} !} \frac{(-i \mathbf{y})^{\mathbf{n}}}{\mathbf{n} !} \int_{\mathbb{R}^{d}} \frac{\mathbf{s}^{\mathbf{k}+\mathbf{n}} d \mathbf{s}}{\left(|\mathbf{s}|^{2 l}+1\right)^{2}}\right\} \\
& +\Theta^{\prime}(\mathbf{x}-\mathbf{y}, t) .
\end{aligned}
$$

It is easy to check that

$$
C(t, \mathbf{s}, \mathbf{x}) \leq \begin{cases}C_{1}(t|\mathbf{s}||\mathbf{x}|)^{2 l-d+2}, & \text { if } t|\mathbf{s}||\mathbf{x}| \leq 1 \\ C_{1}(t|\mathbf{s}||\mathbf{x}|)^{2 l-d}, & \text { if } t|\mathbf{s}||\mathbf{x}|>1\end{cases}
$$


Using this estimate, we obtain from (4.30):

$$
\begin{gathered}
|I(\mathbf{x}, t)| \leq(t|\mathbf{x}|)^{2 l-d+2} \sigma_{d} \int_{0}^{(t|\mathbf{x}|)^{-1}} \frac{r^{2 l+1} d r}{\left(r^{2 l}+1\right)^{2}} \\
\left|I_{\infty}(\mathbf{x}, t)\right| \leq(t|\mathbf{x}|)^{2 l-d} \sigma_{d} \int_{(t|\mathbf{x}|)^{-1}}^{\infty} \frac{r^{2 l-1} d r}{\left(r^{2 l}+1\right)^{2}}=\frac{(t|\mathbf{x}|)^{2 l-d} \sigma_{d}}{2 l} \frac{(t|\mathbf{x}|)^{2 l}}{(t|\mathbf{x}|)^{2 l}+1} .
\end{gathered}
$$

Take $t_{0}>0$. In view of (4.27), (4.28) and (4.29), we have for $t \in\left(0, t_{0}\right)$ :

$$
\begin{array}{r}
K\left(\mathbf{x}-\mathbf{y},-t^{2 l}\right)=K\left(\mathbf{x}-\mathbf{y},-t_{0}^{2 l}\right)-\int_{t}^{t_{0}} K^{\prime}(\mathbf{x}-\mathbf{y}, \xi) d \xi= \\
=F\left(\mathbf{x}, \mathbf{y},-t^{2 l}\right)+\Theta\left(\mathbf{x}-\mathbf{y},-t^{2 l}\right),
\end{array}
$$

where $F(\mathbf{x}, \mathbf{y}, \lambda)$ is defined by $(4.21)$,

$$
\begin{gathered}
\Theta(\mathbf{x}, \lambda)=\tilde{I}(\mathbf{x}, t)+\tilde{I}_{\infty}(\mathbf{x}, t)-\tilde{F}(\mathbf{x})+K\left(\mathbf{x},-t_{0}^{2 l}\right), \\
\tilde{I}(\mathbf{x}, t)=2 l \int_{t}^{t_{0}} \frac{I(\mathbf{x}, \xi) d \xi}{\xi^{2 l-d+1}}, \\
\tilde{I}_{\infty}(\mathbf{x}, t)=2 l \int_{t}^{t_{0}} \frac{I_{\infty}(\mathbf{x}, \xi) d \xi}{\xi^{2 l-d+1}}
\end{gathered}
$$

and

$$
\begin{array}{r}
\tilde{F}(\mathbf{x})=\frac{2 l}{(2 \pi)^{\frac{d}{2}}} \sum_{k=0}^{2 l-d-1} \frac{t_{0}^{k+d-2 l}}{k !(k+d-2 l)} \int_{\mathbb{R}^{d}} \frac{(i \mathbf{s} \cdot \mathbf{x})^{k} d \mathbf{s}}{\left(|\mathbf{s}|^{2 l}+1\right)^{2}}+ \\
+\frac{\ln t_{0}}{(2 \pi)^{\frac{d}{2}}(2 l-d) !} \int_{\mathbb{R}^{d}} \frac{(i \mathbf{s} \cdot \mathbf{x})^{2 l-d} d \mathbf{s}}{\left(|\mathbf{s}|^{2 l}+1\right)^{2}} .
\end{array}
$$

Observe that, in view of (4.33), $F(\mathbf{x}, \mathbf{y}, \lambda)$ can be written also in the form (4.22). From (4.38) and (4.34) we obtain for $t \in\left(0, t_{0}\right)$ changing the order of the integration:

$$
\begin{aligned}
|\tilde{I}(\mathbf{x}, t)| & \leq 2 l|\mathbf{x}|^{2 l-d+2} \int_{t}^{t_{0}} \xi d \xi \int_{0}^{(|\mathbf{x}| \xi)^{-1}} \frac{r^{2 l+1} d r}{\left(r^{2 l}+1\right)^{2}} \\
& =l|\mathbf{x}|^{2 l-d+2}\left(\left(t_{0}^{2}-t^{2}\right) \int_{0}^{\left(|\mathbf{x}| t_{0}\right)^{-1}} \frac{r^{2 l+1} d r}{\left(r^{2 l}+1\right)^{2}}\right. \\
& \left.+\int_{\left(|\mathbf{x}| t_{0}\right)^{-1}}^{(|\mathbf{x}| t)^{-1}} \frac{r^{2 l+1}}{\left(r^{2 l}+\right)^{2}}\left((|\mathbf{x}| r)^{-2}-t^{2}\right) d r\right) \\
& =l|\mathbf{x}|^{2 l-d+2}\left(t_{0}^{2} \int_{0}^{\left(|\mathbf{x}| t_{0}\right)^{-1}} \frac{r^{2 l+1} d r}{\left(r^{2 l}+1\right)^{2}}\right. \\
& \left.-t^{2} \int_{0}^{(|\mathbf{x}| t)^{-1}} \frac{r^{2 l+1} d r}{\left(r^{2 l}+1\right)^{2}}+|\mathbf{x}|^{-2} \int_{\left(|\mathbf{x}| t_{0}\right)^{-1}}^{(|\mathbf{x}| t)^{-1}} \frac{r^{2 l-1}}{\left(r^{2 l}+\right)^{2}} d r\right) .
\end{aligned}
$$

Carrying out the integration by parts, we obtain:

$$
\int_{0}^{(|\mathbf{x}| t)^{-1}} \frac{r^{2 l+1} d r}{\left(r^{2 l}+1\right)^{2}}=-\frac{(|\mathbf{x}| t)^{-2}}{2 l\left((|\mathbf{x}| t)^{-2 l}+1\right)}+\frac{1}{l} \int_{0}^{(|\mathbf{x}| t)^{-1}} \frac{r d r}{r^{2 l}+1}
$$


Furthermore, we have:

$$
\frac{(|\mathbf{x}| t)^{-2}}{(|\mathbf{x}| t)^{-2 l}+1} \leq(|\mathbf{x}| t)^{-2} \text { and } \int_{0}^{(|\mathbf{x}| t)^{-1}} \frac{r d r}{r^{2 l}+1} \leq \frac{1}{2}(|\mathbf{x}| t)^{-2}
$$

The latter estimates, estimate (4.41) and equality (4.42) imply

$$
|\tilde{I}(\mathbf{x}, t)| \leq C_{1}|\mathbf{x}|^{2 l-d},
$$

where $C_{1}>0$ does not depend on $(\mathbf{x}, t) \in \mathbb{R}^{d} \times\left(0, t_{0}\right)$.

Let us estimate the integral $\tilde{I}_{\infty}(\mathbf{x}, t)$ (defined by (4.39)) using (4.35):

$$
\left.\left|\tilde{I}_{\infty}(\mathbf{x}, t)\right| \leq \sigma_{d}|\mathbf{x}|^{2 l-d} \int_{t}^{t_{0}} \frac{|\mathbf{x}|^{2 l} \xi^{2 l-1} d \xi}{(|\mathbf{x}| \xi)^{2 l}+1}=\frac{\sigma_{d}|\mathbf{x}|^{2 l-d}}{2 l} \ln (|\mathbf{x}| \xi)^{2 l}+1\right)\left.\right|_{t} ^{t_{0}}
$$

Then for a small enough $t_{0}$ and $t \in\left(0, t_{0}\right)$ we obtain:

$$
\left|\tilde{I}_{\infty}(\mathbf{x}, t)\right| \leq C_{2}|\mathbf{x}|^{2 l-d}(1+\ln (|\mathbf{x}|+1)),
$$

where $C_{2}>0$ does not depend on $\mathbf{x}, t$.

Observe that

$$
\left|K\left(\mathbf{x},-t_{0}^{2 l}\right)\right|=\left|(2 \pi)^{-\frac{d}{2}} \int_{\mathbb{R}^{d}} \frac{\exp (i \mathbf{p} \cdot \mathbf{x}) d \mathbf{p}}{|\mathbf{p}|^{2 l}+t_{0}^{2 l}}\right| \leq(2 \pi)^{-\frac{d}{2}} \int_{\mathbb{R}^{d}} \frac{d \mathbf{p}}{|\mathbf{p}|^{2 l}+t_{0}^{2 l}}<\infty,
$$

because $2 l>d$. Furthermore, we have from (4.40):

$$
|\tilde{F}(\mathbf{x})| \leq C_{3}\left(1+|\mathbf{x}|^{2 l-d}\right),
$$

where $C_{3}>0$ does not depend on $\mathbf{x}$. This estimate and estimates (4.43), (4.44) and (4.45) imply estimate (4.23) for the function $\Theta(\mathbf{x}, \lambda)$ defined by (4.37). Furthermore, in view of (4.36), we obtain representation (4.20).

Consider the case $2 l=d$. In the same manner as in the previous case we obtain representation (4.24), in which $F_{1}(\lambda)$ is defined by $(4.25)$,

$$
\Theta_{1}(\mathbf{x})=K\left(\mathbf{x},-t_{0}^{2 l}\right), \quad \Theta_{2}(\mathbf{x}, \lambda)=\tilde{I}(\mathbf{x}, t)+\tilde{I}_{\infty}(\mathbf{x}, t)-\tilde{F}(\mathbf{x}),
$$

where $\tilde{I}(\mathbf{x}, t), \tilde{I}_{\infty}(\mathbf{x}, t)$ are defined by (4.38), (4.39), (4.30), (4.31), (4.32) with $k=$ $2 l-d$ and

$$
\tilde{F}(\mathbf{x})=\ln t_{0}(2 \pi)^{-\frac{d}{2}} \int_{\mathbb{R}^{d}} \frac{d \mathbf{s}}{\left(|\mathbf{s}|^{2 l}+1\right)^{2}} .
$$

In the same manner as above, we obtain estimate (4.26). We have only to justify the possibility of differentiation under integral sign in (4.27) and of reconstruction of $K\left(\mathbf{x},-t^{2 l}\right)$ by formula (4.36), because for $2 l=d$ this integral does not converges absolutely. This possibility follows from the obvious membership

$$
\frac{1}{|\mathbf{p}|^{2 l}+t^{2 l}} \in C^{1}\left((0, \infty), L_{2}\left(R^{d}\right)\right)
$$

and from the fact that the Fourier transform on $\mathbb{R}^{d}$ is an isometric linear automorphism of the space $L_{2}\left(\mathbb{R}^{d}\right)$. The lemma is proven. 
Remark 4.7. For the numbers $\eta_{\mathbf{k}}$, defined by

$$
\eta_{\mathbf{k}}=(2 \pi)^{-d} \int_{\mathbb{R}^{d}} \frac{\mathbf{s}^{\mathbf{k}} d \mathbf{s}}{\left(|\mathbf{s}|^{2 l}+1\right)^{2}} \quad(|\mathbf{k}|<4 l-d)
$$

assertions (i) and (ii) of Proposition 4.5 are valid. By a standard method it is possible to show that if $\mathbf{k}=2 \mathbf{m}$ with $\mathbf{m}=\left(m_{1}, m_{2}, \ldots, m_{d}\right) \in \mathbb{Z}_{+}^{d}$, then

$$
\eta_{\mathbf{k}}=(2 \pi)^{-d}=(2 \pi)^{-d} \times \begin{cases}\frac{\left(1-\frac{\frac{d}{2}+|\mathbf{m}|}{l}\right) \pi}{l \sin \left(\frac{\pi}{l}\left(\frac{d}{2}+|\mathbf{m}|\right)\right)} \frac{\prod_{j=1}^{d} \Gamma\left(m_{j}+\frac{1}{2}\right)}{\Gamma\left(\frac{d}{2}+|\mathbf{m}|\right)}, & \text { if }|\mathbf{k}|<2 l-d, \\ \frac{\prod_{j=1}^{d} \Gamma\left(m_{j}+\frac{1}{2}\right)}{\Gamma\left(\frac{d}{2}+|\mathbf{m}|\right) l}, & \text { if }|\mathbf{k}|=2 l-d .\end{cases}
$$

We now turn to the main statement of this subsection.

Proposition 4.8. Assume that $d$ is even, $2 l \geq d$ and the potential $V(\mathbf{x})$ satisfies the additional condition:

$$
\int_{\mathbb{R}^{d}}|\mathbf{x}|^{2(2 l-d)}(\ln (1+|\mathbf{x}|))^{2} V(\mathbf{x}) d \mathbf{x}<\infty .
$$

Then the Birman-Schwinger operator

$$
X_{V}(\lambda)=V^{\frac{1}{2}} R_{\lambda}\left(H_{0}\right) V^{\frac{1}{2}}
$$

of the operator $H_{\gamma}$ has the representation for $\lambda<0$ :

$$
X_{V}(\lambda)=\Phi(\lambda)+T(\lambda),
$$

where $\Phi(\lambda)$ is an integral operator with the kernel

$$
\Phi(\mathbf{x}, \mathbf{y}, \lambda)=(2 \pi)^{-\frac{d}{2}}(V(\mathbf{x}))^{\frac{1}{2}} F(\mathbf{x}, \mathbf{y}, \lambda)(V(\mathbf{y}))^{\frac{1}{2}},
$$

$F(\mathbf{x}, \mathbf{y}, \lambda)$ is defined by (4.21), $T(\lambda)$ is an operator belonging to the Hilbert-Schmidt class $\mathcal{S}_{2}$ and its Hilbert-Schmidt norm is bounded uniformly with respect to a small enough $\lambda<0$, that is

$$
\exists \bar{T}>0, \delta>0: \quad\|T(\lambda)\|_{2} \leq \bar{T} \quad \forall \lambda \in(-\delta, 0) .
$$

Proof. Consider the case $2 l>d$. Let $\Theta(\mathbf{x}, \lambda)$ be the function used in representation (4.20) of the kernel $K(\mathbf{x}-\mathbf{y}, \lambda)$ of the integral operator $(2 \pi)^{\frac{d}{2}} R_{\lambda}\left(H_{0}\right)$. Then we should show that for a small enough $\lambda<0$ the integral operator $T(\lambda)$ with the kernel

$$
T(\mathbf{x}, \mathbf{y}, \lambda)=(2 \pi)^{-\frac{d}{2}}(V(\mathbf{x}))^{\frac{1}{2}} \Theta(\mathbf{x}-\mathbf{y}, \lambda)(V(\mathbf{y}))^{\frac{1}{2}}
$$

has the desired property. In view of estimate (4.23), it is enough to show that the kernel

$$
(V(\mathbf{x}))^{\frac{1}{2}}\left(1+|\mathbf{x}-\mathbf{y}|^{2 l-d}\right)(1+\ln (1+|\mathbf{x}-\mathbf{y}|))(V(\mathbf{y}))^{\frac{1}{2}}
$$

belongs to the Hilbert-Schmidt class. We have:

$$
\int_{\mathbb{R}^{d}} V(\mathbf{x}) d \mathbf{x} \int_{\mathbb{R}^{d}}\left(1+|\mathbf{x}-\mathbf{y}|^{2 l-d}\right)^{2}(1+\ln (1+|\mathbf{x}-\mathbf{y}|))^{2} V(\mathbf{y}) d \mathbf{y} \leq
$$




$$
\int_{\mathbb{R}^{d}} V(\mathbf{x}) d \mathbf{x} \int_{\mathbb{R}^{d}}\left(1+|\mathbf{x}-\mathbf{y}|^{2 l-d}\right)^{2}(1+\ln (1+|\mathbf{x}|)+1+\ln (1+|\mathbf{y}|))^{2} V(\mathbf{y}) d \mathbf{y} .
$$

Then, in view of condition (4.47), we obtain the desired property of the operator $T(\lambda)$.

We now turn to the case $2 l=d$. Let $\Theta_{1}(\mathbf{x}), \Theta_{2}(\mathbf{x}, \lambda)$ be the functions used in representation (4.24) of the kernel $K(\mathbf{x}-\mathbf{y}, \lambda)$. We should show that the integral operator $T_{1}$ with the kernel

$$
T_{1}(\mathbf{x}, \mathbf{y})=(2 \pi)^{-\frac{d}{2}}(V(\mathbf{x}))^{\frac{1}{2}} \Theta_{1}(\mathbf{x}-\mathbf{y})(V(\mathbf{y}))^{\frac{1}{2}}
$$

belongs to the Hilbert-Schmidt class $\mathcal{S}_{2}$, for a small enough $\lambda<0$ the integral operator with the kernel

$$
T_{2}(\mathbf{x}, \mathbf{y}, \lambda)=(2 \pi)^{-\frac{d}{2}}(V(\mathbf{x}))^{\frac{1}{2}} \Theta_{2}(\mathbf{x}-\mathbf{y}, \lambda)(V(\mathbf{y}))^{\frac{1}{2}}
$$

belongs to the class $\mathcal{S}_{2}$ too and its Hilbert-Schmidt norm is bounded uniformly with respect to $\lambda \in(-\delta, 0)$ for some $\delta>0$. The operator $T_{2}(\lambda)$ is treated in the same manner as the operator $T(\lambda)$. Consider the operator $T_{1}$. Observe that, in view of conditions (4.1) and (4.3), the potential $V(\mathbf{x})$ is bounded on $\mathbb{R}^{d}$. Then we have, taking into account condition (4.47) and the fact that $\Theta_{1} \in L_{2}\left(\mathbb{R}^{d}\right)$ :

$$
\int_{\mathbb{R}^{d}} V(\mathbf{x}) d \mathbf{x} \int_{\mathbb{R}^{d}} \Theta_{1}^{2}(\mathbf{x}-\mathbf{y}) V(\mathbf{y}) d \mathbf{y} \leq \sup _{\mathbf{x} \in \mathbb{R}^{d}} V(\mathbf{x}) \int_{\mathbb{R}^{d}} \Theta_{1}^{2}(\mathbf{x}) d \mathbf{x} \int_{\mathbb{R}^{d}} V(\mathbf{y}) d \mathbf{y}<\infty .
$$

Thus, $T_{1} \in \mathcal{S}_{2}$.

\section{Asymptotic expansion for the bottom virtual eigen- value}

In this section we obtain asymptotic expansion for the bottom virtual eigenvalue of the operator $H_{\gamma}$ with respect to a small coupling constant $\gamma<0$. To this end we carry out an asymptotic expansion for the maximal eigenvalue $\tilde{\mu}_{0}(\lambda)$ of the finite-rank portion $\Phi(\lambda)$, extracted from the Birman-Schwinger operator $X_{V}(\lambda)$ in Section 4 . In the case of $\mathbb{R}^{d}$ with $d$ odd $\tilde{\mu}_{0}\left(-t^{2 l}\right)$ admits expansion in a rational Laurent series near the point $t=0$, because the operator function $\Phi\left(-t^{2 l}\right)$ is meromorphic. Hence the asymptotic expansion of the bottom virtual eigenvalue has a power form for an odd $d$. In the case of $\mathbb{R}^{d}$ with $d$ even the operator function $\Phi\left(-t^{2 l}\right)$ is not meromorphic, because it contains summands with $\ln \left(\frac{1}{t}\right)$ in its expansion near the point $t=0$. Hence the asymptotic expansion of the bottom virtual eigenvalue is more complicated in this case.

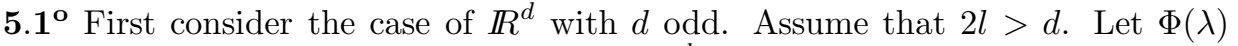
$(\lambda<0)$ be the integral operator acting in $L_{2}\left(\mathbb{R}^{d}\right)$ and having the kernel of the form (4.17), where $F(\mathbf{x}, \mathbf{y}, \lambda)$ is defined by (4.7). In other words, $\Phi(\lambda)$ has the form:

$$
\Phi(\lambda)=\sum_{\mathbf{k}, \mathbf{n}:|\mathbf{k}+\mathbf{n}| \leq 2 m}|\lambda|^{\frac{|\mathbf{k}+\mathbf{n}|-2 m-1}{2 l}} \xi_{\mathbf{k}+\mathbf{n}}\left(\cdot, h_{\mathbf{n}}\right) h_{\mathbf{k}},
$$

where

$$
m=l-\frac{d+1}{2}
$$


$\xi_{\mathbf{k}}$ is defined by $(4.19)$ and

$$
h_{\mathbf{k}}(\mathbf{x})=\frac{(i \mathbf{x})^{\mathbf{k}}}{\mathbf{k} !}(V(\mathbf{x}))^{\frac{1}{2}}(|\mathbf{k}| \leq 2 m) .
$$

Observe that if the potential $V(\mathbf{x})$ satisfies condition (4.16), then the functions $h_{\mathbf{k}}(\mathbf{x})(|\mathbf{k}| \leq 2 m)$ belong to the space $L_{2}\left(\mathbb{R}^{d}\right)$. Taking into account that, by Proposition $4.5, \xi_{\mathbf{k}}=0$ if at least one component of the multi-index $\mathbf{k}$ is odd, we can write formula (5.1) in another form:

$$
\Phi(\lambda)=\frac{1}{|\lambda|^{\frac{m+\frac{1}{2}}{l}}} \sum_{j=0}^{m}|\lambda|^{\frac{j}{l}} \Phi_{j}
$$

and

$$
\Phi_{j}=\sum_{\mathbf{k}, \mathbf{n}:|\mathbf{k}+\mathbf{n}|=2 j} \xi_{\mathbf{k}+\mathbf{n}}\left(\cdot, h_{\mathbf{n}}\right) h_{\mathbf{k}}(j \in\{0,1, \ldots, m\}) .
$$

The following statement about an asymptotic expansion of the maximal eigenvalue $\tilde{\mu}_{0}(\lambda)$ of the operator $\Phi(\lambda)$ is valid:

Lemma 5.1. Assume that $d$ is odd, the number $m$, defined by (5.2), is positive and in addition to conditions (4.1)-(4.3) the potential $V(\mathbf{x})$ is not identically zero and satisfies condition (4.16). Then for $\lambda \uparrow 0$ the following asymptotic expansion holds for the maximal eigenvalue $\tilde{\mu}_{0}(\lambda)$ of the operator $\Phi(\lambda)$ :

$$
\tilde{\mu}_{0}(\lambda)=\frac{\nu_{0}}{|\lambda|^{\frac{m+\frac{1}{2}}{l}}}+\frac{\nu_{1}}{|\lambda|^{\frac{m-\frac{1}{2}}{l}}}+\frac{\nu_{2}}{|\lambda|^{\frac{m-\frac{3}{2}}{l}}}+\cdots+\frac{\nu_{m}}{|\lambda|^{\frac{1}{2 l}}}+O\left(|\lambda|^{\frac{1}{2 t}}\right),
$$

where the numbers $\nu_{k}(k=0,1, \ldots, m)$ have the form:

$$
\begin{gathered}
\nu_{0}=\xi_{\mathbf{o}}\left\|h_{\mathbf{o}}\right\|^{2}=\xi_{\mathbf{0}} \int_{\mathbb{R}^{d}} V(\mathbf{s}) d \mathbf{s}, \\
\nu_{1}=\left(\Phi_{1} X_{0}, X_{0}\right), \\
X_{0}=\frac{h_{\mathbf{0}}}{\left\|h_{\mathbf{o}}\right\|}=\frac{\sqrt{V(\mathbf{x})}}{\sqrt{\int_{\mathbb{R}^{d}} V(\mathbf{s}) d \mathbf{s}}}, \\
\nu_{2}=\left(\Phi_{2} X_{0}, X_{0}\right)+\left(\Phi_{1} X_{1}, X_{0}\right), \\
X_{1}=-\frac{1}{\nu_{0}}\left(\nu_{1} X_{0}-\Phi_{1} X_{0}\right), \\
\nu_{j}=\left(\Phi_{j} X_{0}, X_{0}\right)+\left(\Phi_{j-1} X_{1}, X_{0}\right)+\cdots+\left(\Phi_{1} X_{j-1}, X_{0}\right), \\
X_{j-1}=-\frac{1}{\nu_{0}}\left(\nu_{1} X_{j-2}+\nu_{2} X_{j-3}+\cdots+\nu_{j-1} X_{0}-\Phi_{1} X_{j-2}\right. \\
\left.-\Phi_{2} X_{j-3}-\cdots-\Phi_{j-1} X_{0}\right),
\end{gathered}
$$




$$
\begin{aligned}
\nu_{m}= & \left(\Phi_{m} X_{0}, X_{0}\right)+\left(\Phi_{m-1} X_{1}, X_{0}\right)+\cdots+\left(\Phi_{1} X_{m-1}, X_{0}\right), \\
X_{m-1} & =-\frac{1}{\nu_{0}}\left(\nu_{1} X_{m-2}+\nu_{2} X_{m-3}+\cdots+\nu_{m-1} X_{0}-\Phi_{1} X_{m-2}\right. \\
& \left.-\Phi_{2} X_{m-3}-\cdots-\Phi_{m-1} X_{0}\right)
\end{aligned}
$$

and the operators $\Phi_{j}$ are defined by (5.5).

Proof. Denote $t=|\lambda|^{\frac{1}{t}}$ and consider the polynomial operator function:

$$
\Psi(t)=t^{m+\frac{1}{2}} \Phi\left(-t^{l}\right)=\Phi_{0}+\sum_{j=1}^{m} t^{j} \Phi_{j} .
$$

In view of (5.5), the operators $\Phi_{j}$ are bounded, self-adjoint and have a finite rank. By Rellich Theorem ([Bau], Ch. 3, $n^{o} 3.5 .3$, Theorem 4), the branches of eigenvalues $\mu_{k}(t)$ of the operator $\Psi(t)$ can be chosen to be analytic in a neighborhood of $t=0$ and they are connected with the branches $\tilde{\mu}_{k}(\lambda)$ of eigenvalues of the operator $\Phi(\lambda)$ via the formula:

$$
\tilde{\mu}_{k}(\lambda)=\frac{1}{|\lambda|^{\frac{m+\frac{1}{2}}{l}}} \mu_{k}\left(|\lambda|^{\frac{1}{\tau}}\right) \text {. }
$$

Observe that

$$
\Psi(0)=\Phi_{0}=\xi_{\mathbf{0}}\left(\cdot, h_{\mathbf{o}}\right) h_{\mathbf{0}}
$$

hence the operator $\Psi(0)$ has a unique non-zero eigenvalue $\nu_{0}$, defined by (5.7), this eigenvalue is positive and simple and the normalized eigenvector $X_{0}$, defined by (5.9), corresponds to $\nu_{0}$. Hence the analytic branch $\mu_{0}(t)$ of eigenvalues of $\Psi(t)$, having the property $\mu_{0}(0)=\nu_{0}$, is a maximal eigenvalue of $\Psi(t)$ for a small enough $t \in \mathbb{R}$. Therefore

$$
\tilde{\mu}_{0}(\lambda)=\frac{1}{|\lambda|^{\frac{m+\frac{1}{2}}{l}}} \mu_{0}\left(|\lambda|^{\frac{1}{l}}\right) .
$$

is a maximal eigenvalue of the operator $\Phi(\lambda)$ for a small enough $\lambda<0$. Taking into account that for a small enough $t \mu_{0}(t)$ is a simple eigenvalue of the operator $\Psi(t)$, we can find $\mu_{0}(t)$ making use of a simple version of the method of power series of E. Schrödinger ([Bau], Ch. 3, $n^{o}$ 3.1.2). We search for $\mu_{0}(t)$ and the corresponding eigenvector $X(t)$ in the form:

$$
\begin{gathered}
\mu_{0}(t)=\nu_{0}+\nu_{1} t+\nu_{2} t^{2}+\ldots, \\
X(t)=X_{0}+t X_{1}+t^{2} X_{2}+\ldots,
\end{gathered}
$$

imposing the following normalization condition:

$$
\left(X(t), X_{0}\right)=1
$$

Since $\left\|X_{0}\right\|=1$, the latter condition is equivalent to the following one:

$$
X_{k} \perp X_{0} \quad \forall k \in \mathbb{N} .
$$

Substituting the above series into the equation

$$
\Psi(t) X(t)=\mu_{0}(t) X(t)
$$


taking into account (5.16) and comparing the coefficients near the same powers of $t$, we obtain the equations:

$$
\begin{gathered}
\Phi_{0} X_{0}=\nu_{0} X_{0} \\
\Phi_{0} X_{1}-\nu_{0} X_{1}=\nu_{1} X_{0}-\Phi_{1} X_{0}, \\
\Phi_{0} X_{2}-\nu_{0} X_{2}=\nu_{1} X_{1}+\nu_{2} X_{0}-\Phi_{1} X_{1}-\Phi_{2} X_{0},
\end{gathered}
$$

Already we have found $\nu_{0}$ and $X_{0}$. Let us find $\nu_{1}$ and $X_{1}$. Observe that, since $\operatorname{Im}\left(\Phi_{0}-\nu_{0} I\right)=X_{0}^{\perp}$, equation (5.20) has a solution if and only if

$$
\nu_{1} X_{0}-\Phi_{1} X_{0} \perp X_{0},
$$

that is $\nu_{1}$ is expressed by the formula (5.8). In view of (5.19), we search for the vector $X_{1}$ in the subspace $X_{0}^{\perp}$. Taking into account that, in view of (5.17),

$$
\forall X \in X_{0}^{\perp}: \Phi_{0} X-\nu_{0} X=-\nu_{0} X,
$$

we obtain from (5.20) the expression (5.11) for $X_{1}$. Analogously, equation (5.21) is solvable if and only if

$$
\nu_{1} X_{1}+\nu_{2} X_{0}-\Phi_{1} X_{1}-\Phi_{2} X_{0} \perp X_{0}
$$

that is we get the expression (5.10) for $\nu_{2}$. Furthermore, as above, we obtain from (5.21) the corresponding expression for $X_{2}$. Proceeding with this process and taking into account (5.18), we obtain all the desired formulas. The lemma is proven.

Since in the sequel we shall invert the asymptotic expansion of the form (5.6), we need the following

Lemma 5.2. Consider the equation

$$
t=\epsilon \theta(t)
$$

where

$$
\theta(t)=\left(\nu_{0}+\nu_{1} t+\cdots+\nu_{m} t^{m}+\psi t^{m+\frac{1}{2}}\right)^{\frac{2}{2 m+1}},
$$

$m \in \mathbb{N}$ and $\nu_{k}, \psi \in \mathbb{R}, \nu_{0}>0$. Let $\left\{t_{j}(\epsilon)\right\}_{j=0}^{m+1}$ be a sequence of functions defined by the following recurrence relations:

$$
t_{0}(\epsilon)=0, \quad t_{j}(\epsilon)=\epsilon\left(\theta_{j-1}\left(t_{j-1}(\epsilon)\right)\right)_{j-1},
$$

where

$$
\theta_{j}(t)=\left(\nu_{0}+\nu_{1} t+\cdots+\nu_{j} t^{j}\right)^{\frac{2}{2 m+1}}
$$

Then:

(i) Equation (5.22) has a unique solution $t=t(\epsilon)$ for $\epsilon$ belonging to a neighborhood $\mathcal{O}(0)$ of $\epsilon=0$ and this solution is continuous in $\mathcal{O}(0)$;

(ii) For any $j \in\{0,1, \ldots, m\}$

$$
t(\epsilon)-t_{j}(\epsilon)=O\left(\epsilon^{j+1}\right)
$$

and

$$
t(\epsilon)-t_{m+1}(\epsilon)=O\left(\epsilon^{m+\frac{3}{2}}\right) .
$$


Proof. As is easily seen, the function $\theta(t)$ defined by (5.23) satisfies Lipschitz condition in any neighborhood $(-\delta, \delta)$ :

$$
\forall \delta>0 \exists L_{\delta}>0 \forall t_{1}, t_{2} \in(-\delta, \delta):\left|\theta\left(t_{1}\right)-\theta\left(t_{1}\right)\right| \leq L_{\delta}\left|t_{1}-t_{2}\right| .
$$

This circumstance and the form of the equation (5.22) imply assertion (i) of the lemma and the fact that the successive approximations

$$
\tilde{t}_{0}(t)=0, \tilde{t}_{1}(t)=\epsilon \theta\left(\tilde{t}_{0}(t)\right), \ldots, \tilde{t}_{j}(t)=\epsilon \theta\left(\tilde{t}_{j-1}(t)\right), \ldots
$$

converge to the solution $t(\epsilon)$ of this equation uniformly in some neighborhood $\mathcal{O}(0)$ of the point $\epsilon=0$. Making use of (5.28), we have:

$$
\left|\tilde{t}_{k+1}(\epsilon)-\tilde{t}_{k}(\epsilon)\right| \leq L^{k} \epsilon^{k+1}
$$

hence

$$
\left|t(\epsilon)-\tilde{t}_{j}(\epsilon)\right| \leq \sum_{k=j+1}^{\infty}\left|\tilde{t}_{k+1}(\epsilon)-\tilde{t}_{k}(\epsilon)\right| \leq L^{j} \epsilon^{j+1}\left(1+L \epsilon+L^{2} \epsilon^{2}+\ldots\right) .
$$

This means that

$$
t(\epsilon)-\tilde{t}_{j}(\epsilon)=O\left(\epsilon^{j+1}\right) .
$$

Let us return to the sequence $t_{j}(\epsilon)$, defined by (5.24). Our immediate goal is to prove that

$$
\forall j \in\{0,1, \ldots, m\}: t_{j}(\epsilon)-\tilde{t}_{j}(\epsilon)=O\left(\epsilon^{j+1}\right) .
$$

Let us make use of the induction method. Recall that $t_{0}(\epsilon)=\tilde{t}_{0}(\epsilon)=0$. Assume that (5.31) is valid for some $j \in\{0,1, \ldots, m-1\}$ and prove it for $j$ replaced by $j+1$. Taking into account (5.24 and (5.29), we have:

$$
\begin{aligned}
\left|\tilde{t}_{j+1}(\epsilon)-t_{j+1}(\epsilon)\right| & \leq \epsilon\left\{\left|\theta\left(\tilde{t}_{j}(\epsilon)\right)-\theta\left(t_{j}(\epsilon)\right)\right|+\right. \\
\left|\theta\left(t_{j}(\epsilon)\right)-\theta_{j}\left(t_{j}(\epsilon)\right)\right| & \left.+\left|\theta_{j}\left(t_{j}(\epsilon)\right)-\left(\theta_{j}\left(t_{j}(\epsilon)\right)\right)_{j}\right|\right\} .
\end{aligned}
$$

The property (5.28) and the assumption of the induction imply:

$$
\theta\left(\tilde{t}_{j}(\epsilon)\right)-\theta\left(t_{j}(\epsilon)\right)=O\left(\tilde{t}_{j}(\epsilon)-t_{j}(\epsilon)\right)=O\left(\epsilon^{j+1}\right) .
$$

Observe that, in view of (5.24) and the fact that $\nu_{0}>0$, the functions $t_{j}(\epsilon)$ have the form:

$$
t_{j}(\epsilon)=\sigma_{0} \epsilon+O\left(\epsilon^{2}\right) \quad\left(\sigma_{0}>0, j=0,1, \ldots, m+1\right) .
$$

Taking into account this circumstance and (5.23), (5.25), let us estimate for $j \in$ $\{0,1, \ldots, m-1\}$ :

$$
\begin{aligned}
& \theta\left(t_{j}(\epsilon)\right)-\theta_{j}\left(t_{j}(\epsilon)\right)= \\
& \quad\left(\nu_{0}+\nu_{1} t_{j}(\epsilon)+\cdots+\nu_{j}\left(t_{j}(\epsilon)\right)^{j}+\nu_{j+1}\left(t_{j}(\epsilon)\right)^{j+1}+\cdots+\psi\left(t_{j}(\epsilon)\right)^{m+\frac{1}{2}}\right)^{\frac{2}{2 m+1}}- \\
& \quad\left(\nu_{0}+\nu_{1} t_{j}(\epsilon)+\cdots+\nu_{j}\left(t_{j}(\epsilon)\right)^{j}\right)^{\frac{2}{2 m+1}}=O\left(\left(t_{j}(\epsilon)\right)^{j+1}\right)=O\left(\epsilon^{j+1}\right) .
\end{aligned}
$$

Furthermore, by the sense of the notation $(\ldots)_{j}$,

$$
\theta_{j}\left(t_{j}(\epsilon)\right)-\left(\theta_{j}\left(t_{j}(\epsilon)\right)\right)_{j}=O\left(\epsilon^{j+1}\right) .
$$


The latter estimate and estimates (3.9), (5.35) and (5.32) imply that

$$
t_{j+1}(\epsilon)-\tilde{t}_{j+1}(\epsilon)=O\left(\epsilon^{j+2}\right) .
$$

So, we have proved estimate (5.31), which together with estimate (5.30) yields the desired estimate (5.26).

Let us prove estimate (5.27). Observe that estimate (5.32) is valid also for $j=m$, that is

$$
\begin{aligned}
\left|\tilde{t}_{m+1}(\epsilon)-t_{m+1}(\epsilon)\right| & \leq \epsilon\left\{\left|\theta\left(\tilde{t}_{m}(\epsilon)\right)-\theta\left(t_{m}(\epsilon)\right)\right|+\right. \\
\left|\theta\left(t_{m}(\epsilon)\right)-\theta_{m}\left(t_{m}(\epsilon)\right)\right| & \left.+\left|\theta_{m}\left(t_{m}(\epsilon)\right)-\left(\theta_{m}\left(t_{m}(\epsilon)\right)\right)_{m}\right|\right\} .
\end{aligned}
$$

The property (5.28) and estimate (5.31) imply that

$$
\theta\left(\tilde{t}_{m}(\epsilon)\right)-\theta\left(t_{m}(\epsilon)\right)=O\left(\epsilon^{j+1}\right) .
$$

By (5.36) with $j=m$ we get:

$$
\theta_{m}\left(t_{m}(\epsilon)\right)-\left(\theta_{m}\left(t_{m}(\epsilon)\right)\right)_{m}=O\left(\epsilon^{m+1}\right) .
$$

Furthermore, taking into account (5.34) with $j=m$, we have:

$$
\begin{aligned}
& \theta\left(t_{m}(\epsilon)\right)-\theta_{m}\left(t_{m}(\epsilon)\right)= \\
& \quad\left(\nu_{0}+\nu_{1} t_{m}(\epsilon)+\cdots+\nu_{m}\left(t_{m}(\epsilon)\right)^{m}+\psi\left(t_{m}(\epsilon)\right)^{m+\frac{1}{2}}\right)^{\frac{2}{2 m+1}}- \\
& \quad\left(\nu_{0}+\nu_{1} t_{m}(\epsilon)+\cdots+\nu_{m}\left(t_{m}(\epsilon)\right)^{m}\right)^{\frac{2}{2 m+1}}=O\left(\left(t_{m}(\epsilon)\right)^{m+\frac{1}{2}}\right)=O\left(\epsilon^{m+\frac{1}{2}}\right) .
\end{aligned}
$$

The latter estimate, estimates (5.37), (5.38), (5.39) and estimate (5.30) with $j=m+1$ imply the desired estimate (5.27). The lemma is proven.

We now turn to the main result of this subsection.

Theorem 5.3. Assume that $d$ is odd, $2 l>d$, and in addition to conditions (4.1)(4.3) the potential $V(\mathbf{x})$ is not identically zero and satisfies condition (4.16). Denote $m=l-\frac{d+1}{2}$. Then:

(i) If $m=0$, the operator $H_{\gamma}$ has an unique virtual eigenvalue $\lambda_{\mathbf{0}}(\gamma)$ at $\lambda=0$ and the following asymptotic representation is valid for $\gamma \uparrow 0$ :

$$
\lambda_{\mathbf{0}}(\gamma)=-c_{\mathbf{0}}|\gamma|^{2 l}(1+O(\gamma))
$$

where

$$
c_{\mathbf{0}}=\left(\xi_{0} \int_{\mathbb{R}^{d}} V(\mathbf{x}) d \mathbf{x}\right)^{2 l}
$$

and

$$
\xi_{\mathbf{0}}=(2 \pi)^{-d} \int_{\mathbb{R}^{d}} \frac{d \mathbf{s}}{|\mathbf{s}|^{2 l}+1} .
$$

Recall that $\xi_{0}$ is calculated by (4.19) with $\mathbf{k}=\mathbf{0}$;

(ii) If $m>0$, for the bottom virtual eigenvalue $\lambda_{\mathbf{0}}(\gamma)$ of the operator $H_{\gamma}$ at $\lambda=0$ the following asymptotic expansion is valid for $\gamma \uparrow 0$ :

$$
\lambda_{\mathbf{0}}(\gamma)=-|\gamma|^{\frac{2 l}{2 m+1}}\left(\delta_{0}+\delta_{1}|\gamma|^{\frac{2}{2 m+1}}+\cdots+\delta_{m}|\gamma|^{\frac{2 m}{2 m+1}}+O(\gamma)\right)^{l},
$$


where the numbers $\delta_{0}, \delta_{1}, \ldots, \delta_{m}$ are coefficients of a polynomial

$$
p(\epsilon)=\epsilon\left(\delta_{0}+\delta_{1} \epsilon+\cdots+\delta_{m} \epsilon^{m}\right),
$$

which is calculated by the following procedure:

(A) The functions

$$
\theta_{j}(t)=\left(\nu_{0}+\nu_{1} t+\cdots+\nu_{j} t^{j}\right)^{\frac{2}{2 m+1}} \quad(j=0,1, \ldots, m)
$$

are considered, where the numbers $\nu_{0}, \nu_{1}, \ldots, \nu_{m}$ are defined by equalities (5.7)-(5.15), in which the operators $\Phi_{j}$ are defined by (5.5), (5.3) and (4.19);

(B) The polynomials $t_{j}(\epsilon)(j=0,1,2, \ldots, m+1)$ are constructed in the following manner:

$$
t_{0}(\epsilon)=0, \quad t_{j}(\epsilon)=\epsilon\left(\theta_{j-1}\left(t_{j-1}(\epsilon)\right)\right)_{j-1} \quad(j=1,2, \ldots, m+1)
$$

(C) We put $p(\epsilon)=t_{m+1}(\epsilon)$.

Proof. First we shall prove assertion (ii). Assume that $m>0$. Let $\mu_{0}^{+}(\lambda)$ be the maximal characteristic branch of the operator $H_{0}$ with respect to $V$ in the gap $(-\infty, 0)$ of $\sigma\left(H_{0}\right)$, that is, this is the maximal positive eigenvalue of the Birman-Schwinger operator $X_{V}(\lambda)$ (see Definition 3.2). Let $\tilde{\mu}_{0}(\lambda)$ be the maximal eigenvalue of the finite-rank portion $\Phi(\lambda)$ of the operator $X_{V}(\lambda)$. By Proposition 4.4 and Lemma 3.4, we have for some $\bar{T}>0$ :

$$
\tilde{\mu}_{0}(\lambda)-\bar{T} \leq \mu_{0}^{+}(\lambda) \leq \tilde{\mu}_{0}(\lambda)+\bar{T} \quad(\lambda<0) .
$$

These estimates and the asymptotic expansion (5.6) for $\lambda \uparrow 0$ (Lemma 5.1) imply that for some $\bar{S}>0$ and a small enough $\lambda<0$

$$
\sigma(\lambda)-\bar{S} \leq \mu_{0}^{+}(\lambda) \leq \sigma(\lambda)+\bar{S}
$$

where the function $\sigma(\lambda)$ is defined on $(-\infty, 0)$ and has the form:

$$
\sigma(\lambda)=\frac{\nu_{0}}{|\lambda|^{\frac{m+\frac{1}{2}}{l}}}+\frac{\nu_{1}}{|\lambda|^{\frac{m-\frac{1}{2}}{l}}}+\frac{\nu_{2}}{|\lambda|^{\frac{m-\frac{3}{2}}{l}}}+\cdots+\frac{\nu_{m}}{|\lambda|^{\frac{1}{2 l}}} .
$$

The latter estimates and Proposition 3.16 imply that $\mu_{0}^{+}(\lambda)$ is a main characteristic branch of the operator $H_{0}$, with respect to $\mathrm{V}$, near the endpoint $\lambda=0$ of $(-\infty, 0)$ and, furthermore, for the bottom virtual eigenvalue $\lambda_{\mathbf{0}}(\gamma)$ of $H_{\gamma}$ the estimates are valid for a small enough $\gamma<0$ :

$$
\Lambda_{+}\left(\frac{1}{|\gamma|}\right) \leq \lambda_{\mathbf{0}}(\gamma) \leq \Lambda_{-}\left(\frac{1}{|\gamma|}\right)
$$

where $\Lambda_{+}$and $\Lambda_{-}$are the inverses of the functions $\sigma(\lambda)+\bar{S}$ and $\sigma(\lambda)-\bar{S}$, respectively. In order to find the bounds $\Lambda_{+}\left(\frac{1}{|\gamma|}\right)$ and $\Lambda_{-}\left(\frac{1}{|\gamma|}\right)$ in (5.42), we should solve the equation of the form:

$$
\sigma(\lambda)+\psi=\frac{1}{|\gamma|}
$$


where $\psi$ takes one of the values: $\bar{S}$ or $-\bar{S}$. Denote

$$
t=|\lambda|^{\frac{1}{l}}
$$

and

$$
\epsilon=|\gamma|^{\frac{2}{2 m+1}} .
$$

In view of (5.41), equation (5.43) acquires the form:

$$
t=\epsilon \theta(t),
$$

where

$$
\theta(t)=\left(\nu_{0}+\nu_{1} t+\cdots+\nu_{m} t^{m}+\psi t^{m+\frac{1}{2}}\right)^{\frac{2}{2 m+1}} .
$$

By Lemma 5.2, the solution of equation (5.46) has the form:

$$
t(\epsilon)=p(\epsilon)+O\left(\epsilon^{m+\frac{3}{2}}\right)
$$

where the polynomial $p(\epsilon)$ is calculated according to the procedure (A), (B) and (C). This circumstance, notation (5.44), (5.45) and estimates (5.42) imply the desired asymptotic expansion (5.40).

We now turn to assertion (i) of the theorem. Observe that in the case $m=0$ the finite-rank portion $\Phi(\lambda)$ of the Birman-Schwinger operator $X_{V}(\lambda)$ is of rank one and has the form:

$$
\Phi(\lambda)=\frac{\xi_{\mathbf{0}}}{|\lambda|^{\frac{1}{2 t}}}\left(\cdot, h_{\mathbf{0}}\right) h_{\mathbf{0}}
$$

The latter operator has only one non-zero branch of eigenvalues:

$$
\mu_{\mathbf{0}}(\lambda)=\frac{\xi_{\mathbf{0}}}{|\lambda|^{\frac{1}{2 l}}}\left\|h_{\mathbf{0}}\right\|^{2} .
$$

Using the same arguments, as in the proof of assertion (ii), we prove assertion (i). The theorem is proven.

5.2 $2^{\text {o }}$ We now turn to the case of $\mathbb{R}^{d}$ with $d$ even. Assume that $2 l>d$. Let $\Phi(\lambda)(\lambda<0)$ be the integral operator acting in the space $L_{2}\left(\mathbb{R}^{d}\right)$ and having the kernel $\Phi(\mathbf{x}, \mathbf{y}, \lambda)$, defined by (4.48), where $F(\mathbf{x}, \mathbf{y}, \lambda)$ is defined by (4.21). In other words, $\Phi(\lambda)$ has the form:

$$
\Phi(\lambda)=\sum_{\mathbf{k}, \mathbf{n}:|\mathbf{k}+\mathbf{n}| \leq 2 m-1} \frac{\xi_{\mathbf{k}+\mathbf{n}}}{|\lambda|^{\frac{2 m-\mathbf{k}+\mathbf{n} \mid}{2 l}}}\left(\cdot, h_{\mathbf{n}}\right) h_{\mathbf{k}}+\ln \left(\frac{1}{|\lambda|}\right) \sum_{\mathbf{k}, \mathbf{n}:|\mathbf{k}+\mathbf{n}|=2 m} \eta_{\mathbf{k}+\mathbf{n}}\left(\cdot, h_{\mathbf{n}}\right) h_{\mathbf{k}},
$$

where

$$
m=l-\frac{d}{2},
$$

the functions $h_{\mathbf{k}}(\mathbf{x})$ are defined by (5.3) and the numbers $\xi_{\mathbf{k}}$ and $\eta_{\mathbf{k}}$ are defined by (4.19) and (4.46), respectively. In this subsection we assume that the potential $V(\mathbf{x})$ satisfies condition (4.47), which in particular ensures the membership of the functions 
$h_{\mathbf{k}}(\mathbf{x})(|\mathbf{k}| \leq 2 m)$ to the class $L_{2}\left(\mathbb{R}^{d}\right)$. Taking into account Proposition 4.5 , we can write formula (5.47) in another form:

$$
\Phi(\lambda)=\frac{1}{|\lambda|^{\frac{m}{T}}} \sum_{j=0}^{m-1}|\lambda|^{\frac{j}{l}} \Phi_{j}+\ln \left(\frac{1}{|\lambda|}\right) \Psi_{m},
$$

where

$$
\Phi_{j}=\sum_{\mathbf{k}, \mathbf{n}:|\mathbf{k}+\mathbf{n}|=2 j} \xi_{\mathbf{k}+\mathbf{n}}\left(\cdot, h_{\mathbf{n}}\right) h_{\mathbf{k}}(j \in\{0,1, \ldots, m-1\})
$$

and

$$
\Psi_{m}=\sum_{\mathbf{k}, \mathbf{n}:|\mathbf{k}+\mathbf{n}|=2 m} \eta_{\mathbf{k}+\mathbf{n}}\left(\cdot, h_{\mathbf{n}}\right) h_{\mathbf{k}} .
$$

The following statement about an asymptotic expansion of the maximal eigenvalue $\tilde{\mu}_{0}(\lambda)$ of the operator $\Phi(\lambda)$ is valid:

Lemma 5.4. Assume that $d$ is even, the number $m$, defined by (5.48), is positive and in addition to conditions (4.1)-(4.3) the potential $V(\mathbf{x})$ is not identically zero and satisfies condition (4.47). Then for $\lambda \uparrow 0$ the following asymptotic expansion holds for the maximal eigenvalue $\tilde{\mu}_{0}(\lambda)$ of the operator $\Phi(\lambda)$ :

$$
\tilde{\mu}_{0}(\lambda)=\frac{\nu_{0}}{|\lambda|^{\frac{m}{l}}}+\frac{\nu_{1}}{|\lambda|^{\frac{m-1}{l}}}+\frac{\nu_{2}}{|\lambda|^{\frac{m-2}{l}}}+\cdots+\frac{\nu_{m-1}}{|\lambda|^{\frac{1}{t}}}+\nu_{m} \ln \left(\frac{1}{|\lambda|}\right)+O(1),
$$

where the numbers $\nu_{k}(k=0,1, \ldots, m)$ have the form:

$$
\begin{gathered}
\nu_{0}=\xi_{\mathbf{o}}\left\|h_{\mathbf{o}}\right\|^{2}=\xi_{\mathbf{0}} \int_{\mathbb{R}^{d}} V(\mathbf{s}) d \mathbf{s}, \\
\nu_{1}=\left(\Phi_{1} X_{0}, X_{0}\right), \\
X_{0}=\frac{h_{\mathbf{0}}}{\left\|h_{\mathbf{o}}\right\|}=\frac{\sqrt{V(\mathbf{x})}}{\sqrt{\int_{\mathbb{R}^{d}} V(\mathbf{s}) d \mathbf{s}}}, \\
\nu_{2}=\left(\Phi_{2} X_{0}, X_{0}\right)+\left(\Phi_{1} X_{1}, X_{0}\right), \\
X_{1}=-\frac{1}{\nu_{0}}\left(\nu_{1} X_{0}-\Phi_{1} X_{0}\right), \\
\nu_{j}=\left(\Phi_{j} X_{0}, X_{0}\right)+\left(\Phi_{j-1} X_{1}, X_{0}\right)+\cdots+\left(\Phi_{1} X_{j-1}, X_{0}\right), \\
X_{j-1}=\frac{1}{\nu_{0}}\left(\nu_{1} X_{j-2}\right)+\nu_{2} X_{j-3}+\cdots+\nu_{j-1} X_{0}-\Phi_{1} X_{j-2} \\
\left.-\Phi_{2} X_{j-3}-\cdots-\Phi_{j-1} X_{0}\right), \\
\nu_{m-2}=\left(\Phi_{m-2} X_{0}, X_{0}\right)+\left(\Phi_{m-3} X_{1}, X_{0}\right)+\cdots+\left(\Phi_{1} X_{m-3}, X_{0}\right),
\end{gathered}
$$




$$
\begin{gathered}
X_{m-3}=-\frac{1}{\nu_{0}}\left(\nu_{1} X_{m-4}+\nu_{2} X_{m-5}+\cdots+\nu_{m-3} X_{0}-\Phi_{1} X_{m-4}\right. \\
\left.-\quad \Phi_{2} X_{m-5}-\cdots-\Phi_{m-3} X_{0}\right), \\
\nu_{m-1}=\left(\Phi_{m-1} X_{0}, X_{0}\right)+\left(\Phi_{m-2} X_{1}, X_{0}\right)+\cdots+\left(\Phi_{1} X_{m-2}, X_{0}\right), \\
X_{m-2}=-\frac{1}{\nu_{0}}\left(\nu_{1} X_{m-3}+\nu_{2} X_{m-4}+\cdots+\nu_{m-2} X_{0}-\Phi_{1} X_{m-3}\right. \\
\left.-\quad \Phi_{2} X_{m-4}-\cdots-\Phi_{m-2} X_{0}\right), \\
\nu_{m}=\left(\Psi_{m} X_{0}, X_{0}\right)
\end{gathered}
$$

and the operators $\Phi_{j}$ and $\Psi_{m}$ are defined by (5.50) and (5.51).

Proof. Let us denote

$$
t=|\lambda|^{\frac{1}{\tau}}
$$

and consider the operator function:

$$
\Psi(t)=t^{m} \Phi\left(-t^{l}\right)=\Phi_{0}+\sum_{j=1}^{m-1} t^{j} \Phi_{j}+l t^{m} \ln \left(\frac{1}{t}\right) \Psi_{m} .
$$

Unlike the case of an odd $d$, this function is not a polynomial one. We shall reduce it to a polynomial operator function of two variables $t, v$ putting

$$
v=t \ln \left(\frac{1}{t}\right) .
$$

In other words, we represent:

$$
\Psi(t)=F\left(t, t \ln \left(\frac{1}{t}\right)\right)
$$

where

$$
F(t, v)=\Phi_{0}+\sum_{j=1}^{m-1} t^{j} \Phi_{j}+l t^{m-1} v \Psi_{m}
$$

Our immediate goal is to find a power expansion of the maximal eigenvalue $\omega_{0}(t, v)$ of the operator $F(t, v)$. Observe that

$$
F(0,0)=\Phi_{0}=\xi_{\mathbf{0}}\left(\cdot, h_{\mathbf{0}}\right) h_{\mathbf{0}},
$$

hence the operator $F(0,0)$ has a unique non-zero eigenvalue $\nu_{0}$, defined by $(5.53)$, this eigenvalue is positive and simple and the normalized eigenvector $X_{0}$, defined by (5.55), corresponds to $\nu_{0}$. Hence, as is known (see [Bau], Supplement, S2, Corollary 1 ), there exists a branch $\omega_{0}(t, v)$ of eigenvalues of the operator function $F(t, v)$, such that $\omega_{0}(0,0)=\nu_{0}$ and it is analytic with respect to the variables $t, v$ in a neighborhood $\mathcal{O}(0,0) \subset \mathbb{C}^{2}$ of the point $(0,0)$ and the corresponding branch of normalized eigenvectors $X(t, v)$ is analytic in $\mathcal{O}(0,0)$ too. Furthermore, it is possible to choose $\mathcal{O}(0,0)$ such that $\omega_{0}(t, v)$ is a maximal eigenvalue of the operator $F(t, v)$ for any $(t, v) \in \mathcal{O}(0,0)$. Thus, we can search for $\omega_{0}(t, v)$ and $X(t, v)$ in the form:

$$
\omega_{0}(t, v)=\nu_{0}+\rho_{1}(v) t+\cdots+\rho_{m-2}(v) t^{m-2}+\rho_{m-1}(v) t^{m-1}+t^{m} r(t, v)
$$


and

$$
\omega_{0}(t, v)=\nu_{0}+t X_{1}(v)+\cdots+t^{m-2} X_{m-2}(v)+t^{m-1} X_{m-1}(v)+t^{m} R(t, v),
$$

where the functions $\rho_{j}(v)$ and the vector functions $X_{j}(v)$ are analytic in a neighborhood $\mathcal{O}(0) \subset \mathbb{C}$ and the function $r(t, v)$ and the vector function $X(t, v)$ are analytic in a neighborhood $\mathcal{O}(0,0) \subset \mathbb{C}^{2}$. We impose on $X(t, v)$ also the following normalization condition:

$$
\left(X(t, v), X_{0}\right)=1 \quad \forall(t, v) \in \mathcal{O}(0,0) .
$$

Substituting the expansions (5.69) and (5.70) into the equation

$$
F(t, v) X(t, v)=\omega(t, v) X(t, v),
$$

taking into account (5.68) and comparing the coefficients near the same powers of $t$, we get:

$$
\begin{aligned}
& \Phi_{0} X_{1}(v)-\nu_{0} X_{1}(v)=\rho_{1}(v) X_{0}-\Phi_{1} X_{0}, \\
& \Phi_{0} X_{m-2}(v)-\nu_{0} X_{m-2}(v)=\rho_{1}(v) X_{m-3}(v)+\cdots+\rho_{m-2}(v) X_{0} \\
& \text { - } \Phi_{1} X_{m-3}(v)-\cdots-\Phi_{m-2} X_{0} \text {, } \\
& \Phi_{0} X_{m-1}(v)-\nu_{0} X_{m-1}(v)=\rho_{1}(v) X_{m-2}(v)+\cdots+\rho_{m-1}(v) X_{0} \\
& \text { - } \Phi_{1} X_{m-2}(v)-\cdots-\left(\Phi_{m-1}+l v \Psi_{m}\right) X_{0} \text {. }
\end{aligned}
$$

Making use of the same arguments, as in the proof of Lemma 5.1, we obtain that

$$
\forall j \in\{1,2, \ldots, m-2\}: \rho_{j}(v)=\nu_{j}
$$

and

$$
\rho_{m-1}(v)=\nu_{m-1}+l v \nu_{m}
$$

where the numbers $\nu_{j}(j=1,2, \ldots, m)$ are defined by (5.54)-(5.64). Thus, we get the following expansion:

$$
\omega(t, v)=\nu_{0}+\nu_{1} t+\cdots+\nu_{m-2} t^{m-2}+\nu_{m-1} t^{m-1}+l v \nu_{m} t^{m-1}+t^{m} r(t, v)
$$

from which and (5.65), (5.66), (5.67) and (5.68) we obtain the desired asymptotic expansion (5.52) for the branch $\tilde{\mu}_{0}(\lambda)$ of the maximal eigenvalue of the operator $\Phi(\lambda)$. The lemma is proven.

As in the case of an odd $d$, in the sequel we shall invert the asymptotic expansion of the form (5.52). To this end we need the following lemma, which is analogous to Lemma 5.2:

Lemma 5.5. Consider the equation

$$
t=\epsilon \theta(t)
$$

where

$$
\theta(t)=\left(\nu_{0}+\nu_{1} t+\cdots+\nu_{m-1} t^{m-1}+l \nu_{m} t^{m} \ln \left(\frac{1}{t}\right)+\psi t^{m}\right)^{\frac{1}{m}}
$$


$m \in \mathbb{N}$ and $\nu_{k}, \psi, l \in \mathbb{R}, \nu_{0}>0$. Let $\left\{t_{j}(\epsilon)\right\}_{j=0}^{m+1}$ be a sequence of functions defined by the following recurrence relations:

$$
t_{0}(\epsilon)=0, \quad t_{j}(\epsilon)=\epsilon\left(\theta_{j-1}\left(t_{j-1}(\epsilon)\right)\right)_{j-1}(j=1,2, \ldots, m)
$$

and

$$
t_{m+1}(\epsilon)=\epsilon Q_{m}\left(t_{m}(\epsilon)\right)
$$

where

$$
\theta_{j}(t)=\left(\nu_{0}+\nu_{1} t+\cdots+\nu_{j} t^{j}\right)^{\frac{1}{m}}(j=0,1, \ldots, m-1)
$$

and

$$
\begin{aligned}
Q_{m}\left(t_{m}(\epsilon)\right) & =\left\{\left(\nu_{0}+\nu_{1} t_{m}(\epsilon)+\cdots+\nu_{m-1}\left(t_{m}(\epsilon)\right)^{m-1}\right)_{m-1}\right. \\
& \left.+l \nu_{m}\left(\left(t_{m}(\epsilon)\right)_{1}\right)^{m} \ln \left(\frac{1}{\epsilon}\right)\right\}^{\frac{1}{m}}
\end{aligned}
$$

Then:

(i) Equation (5.46) has a unique solution $t=t(\epsilon)$ for $\epsilon$ belonging to a neighborhood $\mathcal{O}(0)$ of $\epsilon=0$ and this solution is continuous in $\mathcal{O}(0)$;

(ii) For any $j \in\{0,1, \ldots, m-1\}$

$$
t(\epsilon)-t_{j}(\epsilon)=O\left(\epsilon^{j+1}\right)
$$

and

$$
\begin{gathered}
t(\epsilon)-t_{m}(\epsilon)=O\left(\epsilon^{m+1} \ln \left(\frac{1}{\epsilon}\right)\right), \\
t(\epsilon)-t_{m+1}(\epsilon)=O\left(\epsilon^{m+1}\right) .
\end{gathered}
$$

Proof. As is easily seen, the function $\theta(t)$ defined by (5.72) satisfies Lipschitz condition in any neighborhood $(-\delta, \delta)$ :

$$
\forall \delta>0 \exists L_{\delta}>0 \forall t_{1}, t_{2} \in(-\delta, \delta):\left|\theta\left(t_{1}\right)-\theta\left(t_{1}\right)\right| \leq L_{\delta}\left|t_{1}-t_{2}\right| .
$$

This circumstance and the form of the equation (5.46) imply assertion (i) of the lemma.

Along with the functions $t_{j}(\epsilon)$, defined by (5.73) and (5.74), consider the successive approximations

$$
\tilde{t}_{0}(t)=0, \tilde{t}_{1}(t)=\epsilon \theta\left(\tilde{t}_{0}(t)\right), \ldots, \tilde{t}_{j}(t)=\epsilon \theta\left(\tilde{t}_{j-1}(t)\right), \ldots
$$

for the solution of equation (5.46). In the same manner, as in the proof of Lemma 5.2 , we prove the estimates

$$
\begin{gathered}
t(\epsilon)-\tilde{t}_{j}(\epsilon)=O\left(\epsilon^{j+1}\right), \\
t_{j}(\epsilon)-\tilde{t}_{j}(\epsilon)=O\left(\epsilon^{j+1}\right) \text { for } j \in\{0,1, \ldots, m-1\}
\end{gathered}
$$

and estimate (5.77) of assertion (ii) of the proposition. Furthermore, in view of (5.73), (5.74) and the fact that $\nu_{0}>0$, the functions $t_{j}(\epsilon)$ have the form:

$$
t_{j}(\epsilon)=\sigma_{0} \epsilon+O\left(\epsilon^{2}\right) \quad\left(\sigma_{0}>0, j=0,1, \ldots, m+1\right) .
$$


In view of (5.81) and (5.73),

$$
\begin{aligned}
\left|\tilde{t}_{m}(\epsilon)-t_{m}(\epsilon)\right| & \leq \epsilon\left\{\left|\theta\left(\tilde{t}_{m-1}(\epsilon)\right)-\theta\left(t_{m-1}(\epsilon)\right)\right|\right. \\
& +\left|\theta\left(t_{m-1}(\epsilon)\right)-\theta_{m-1}\left(t_{m-1}(\epsilon)\right)\right|+\mid \theta_{m-1}\left(t_{m-1}(\epsilon)\right) \\
& \left.-\left(\theta_{m-1}\left(t_{m-1}(\epsilon)\right)\right)_{m-1} \mid\right\} .
\end{aligned}
$$

By the sense of the notation $(\cdots)_{m-1}$,

$$
\mid \theta_{m-1}\left(t_{m-1}(\epsilon)\right)-\left(\theta_{m-1}\left(t_{m-1}(\epsilon)\right)\right)_{m-1}=O\left(\epsilon^{m}\right) .
$$

Furthermore, in view of (5.80) and estimate (5.83), we have:

$$
\theta\left(\tilde{t}_{m-1}(\epsilon)\right)-\theta\left(t_{m-1}(\epsilon)\right)=O\left(\epsilon^{m}\right) .
$$

Let us estimate, taking into account (5.84):

$$
\begin{aligned}
& \theta\left(t_{m-1}(\epsilon)\right)-\theta_{m-1}\left(t_{m}(\epsilon)\right)= \\
& \quad\left\{\nu_{0}+\nu_{1} t_{m-1}(\epsilon)+\cdots+\nu_{m-1}\left(t_{m-1}(\epsilon)\right)^{m-1}+\right. \\
& \left.\quad l \nu_{m} \ln \left(\frac{1}{t_{m-1}(\epsilon)}\right)\left(t_{m-1}(\epsilon)\right)^{m}+\psi\left(t_{m-1}(\epsilon)\right)^{m}\right\}^{\frac{1}{m}}- \\
& \quad\left\{\nu_{0}+\nu_{1} t_{m-1}(\epsilon)+\cdots+\nu_{m-1}\left(t_{m-1}(\epsilon)\right)^{m-1}\right\}^{\frac{1}{m}}=O\left(\epsilon^{m} \ln \left(\frac{1}{\epsilon}\right)\right) .
\end{aligned}
$$

Estimates (5.85), (5.86), (5.87) and (5.88) imply estimate

$$
\tilde{t}_{m}(\epsilon)-t_{m}(\epsilon)=O\left(\epsilon^{m+1} \ln \left(\frac{1}{\epsilon}\right)\right),
$$

which together with (5.82) implies the desired estimate (5.78).

We now turn to the proof of estimate (5.79). We have, taking into account (5.81) and (5.74):

$$
\begin{aligned}
\left|\tilde{t}_{m+1}(\epsilon)-t_{m+1}(\epsilon)\right| & \leq \epsilon\left\{\left|\theta\left(\tilde{t}_{m}(\epsilon)\right)-\theta\left(t_{m}(\epsilon)\right)\right|+\right. \\
\left|\theta\left(t_{m}(\epsilon)\right)-\theta_{m}\left(t_{m}(\epsilon)\right)\right| & \left.+\left|\theta_{m}\left(t_{m}(\epsilon)\right)-Q_{m}\left(t_{m}(\epsilon)\right)\right|\right\}
\end{aligned}
$$

where

$$
\theta_{m}(t)=\left\{\nu_{0}+\nu_{1} t+\cdots+\nu_{m-1} t^{m-1}+l \nu_{m} \ln \left(\frac{1}{t}\right) t^{m}\right\}^{\frac{1}{m}} .
$$

Making use of property (5.80) and estimate (5.89), we get:

$$
\theta\left(\tilde{t}_{m}(\epsilon)\right)-\theta\left(t_{m}(\epsilon)\right)=O\left(\epsilon^{m+1} \ln \left(\frac{1}{\epsilon}\right)\right) .
$$

Furthermore, taking into account (5.84), we have:

$$
\begin{aligned}
\theta\left(t_{m}(\epsilon)\right)-\theta_{m}\left(t_{m}(\epsilon)\right) & =\left\{\nu_{0}+\nu_{1} t_{m}(\epsilon)+\cdots+\nu_{m-1}\left(t_{m}(\epsilon)\right)^{m-1}+\right. \\
l \nu_{m} \ln \left(\frac{1}{t_{m}(\epsilon)}\right)\left(t_{m}(\epsilon)\right)^{m} & \left.+\psi\left(t_{m}(\epsilon)\right)^{m}\right\}^{\frac{1}{m}}- \\
\left\{\nu_{0}+\nu_{1} t_{m}(\epsilon)\right. & +\cdots+\nu_{m-1}\left(t_{m}(\epsilon)\right)^{m-1} \\
& \left.+l \nu_{m} \ln \left(\frac{1}{t_{m}(\epsilon)}\right)\left(t_{m}(\epsilon)\right)^{m}\right\}^{\frac{1}{m}}=O\left(\epsilon^{m}\right) .
\end{aligned}
$$


Let us estimate the third summand in the right hand side of (5.90). Taking into account definitions (5.91) and (5.76), we get:

$$
\begin{aligned}
\theta_{m}\left(t_{m}(\epsilon)\right)-Q_{m}\left(t_{m}(\epsilon)\right) & =O\left(\epsilon^{m}\right)+O\left(\ln \left(\frac{1}{t_{m}(\epsilon)}\right)\left(t_{m}(\epsilon)\right)^{m}\right. \\
& \left.-\ln \left(\frac{1}{\epsilon}\right)\left(\left(t_{m}(\epsilon)\right)_{1}\right)^{m}\right) .
\end{aligned}
$$

On the other hand, in view of (5.84), we have:

$$
\begin{aligned}
\ln \left(\frac{1}{t_{m}(\epsilon)}\right)\left(t_{m}(\epsilon)\right)^{m} & -\ln \left(\frac{1}{\epsilon}\right)\left(\left(t_{m}(\epsilon)\right)_{1}\right)^{m}=\epsilon^{m} \sigma_{0}^{m}(1+O(\epsilon))\left(\ln \left(\frac{1}{\epsilon}\right)\right. \\
& \left.-\ln \sigma_{0}+O(\epsilon)\right)-\epsilon^{m} \sigma_{0}^{m} \ln \left(\frac{1}{\epsilon}\right)=O\left(\epsilon^{m}\right) .
\end{aligned}
$$

The latter estimate and estimates (5.94), (5.92), (5.93), (5.90) and (5.82) imply the desired estimate (5.79). The lemma is proven.

We now turn to the main result of this subsection.

Theorem 5.6. Assume that $d$ is even, $2 l \geq d$, and in addition to conditions (4.1)(4.3) the potential $V(\mathbf{x})$ is not identically zero and satisfies condition (4.47). Denote $m=l-\frac{d}{2}$. Then:

(i) If $m=0$, the operator $H_{\gamma}$ has an unique virtual eigenvalue $\lambda_{\mathbf{0}}(\gamma)$ at $\lambda=0$ and the following asymptotic representation is valid for $\gamma \uparrow 0$ :

$$
\ln \left(\frac{1}{\left|\lambda_{\mathbf{0}}(\gamma)\right|}\right)=\frac{f}{|\gamma|}(1+O(\gamma))
$$

where

$$
f=\left(\eta_{\mathbf{0}} \int_{\mathbb{R}^{d}} V(\mathbf{x}) d \mathbf{x}\right)^{-1}
$$

and

$$
\eta_{\mathbf{0}}=(2 \pi)^{-d} \int_{\mathbb{R}^{d}} \frac{d \mathbf{s}}{\left(|\mathbf{s}|^{2 l}+1\right)^{2}} .
$$

Recall that $\eta_{\mathbf{0}}$ is calculated by (4.46) with $\mathbf{k}=\mathbf{0}$;

(ii) If $m>0$, for the bottom virtual eigenvalue $\lambda_{\mathbf{0}}(\gamma)$ of the operator $H_{\gamma}$ at $\lambda=0$ the following asymptotic expansion is valid for $\gamma \uparrow 0$ :

$$
\lambda_{\mathbf{0}}(\gamma)=-|\gamma|^{\frac{l}{m}}\left(\eta\left(|\gamma|^{\frac{1}{m}}\right)+O(\gamma)\right)^{l},
$$

where the function $\eta(\epsilon)$ has the form:

$$
\eta(\epsilon)=\left(\delta_{0}+\delta_{1} \epsilon+\cdots+\delta_{m-1} \epsilon^{m-1}+\delta_{m} \epsilon^{m} \ln \left(\frac{1}{\epsilon}\right)\right)^{\frac{1}{m}}
$$

and it is calculated by the following procedure:

(A) The functions

$$
\theta_{j}(t)=\left(\nu_{0}+\nu_{1} t+\cdots+\nu_{j} t^{j}\right)^{\frac{1}{m}} \quad(j=0,1, \ldots, m-1)
$$


are considered, where the numbers $\nu_{0}, \nu_{1}, \ldots, \nu_{m}$ are defined by equalities (5.53)(5.63), in which the operators $\Phi_{j}$ are defined by (5.50), (5.3) and (4.19);

(B) The polynomials $t_{j}(\epsilon)(j=0,1,2, \ldots, m)$ are constructed in the following manner:

$$
t_{0}(\epsilon)=0, \quad t_{j}(\epsilon)=\epsilon\left(\theta_{j-1}\left(t_{j-1}(\epsilon)\right)\right)_{j-1} \quad(j=1,2, \ldots, m) ;
$$

(C) We put

$$
\begin{aligned}
\eta(\epsilon) & =\left\{\left(\nu_{0}+\nu_{1} t_{m}(\epsilon)+\cdots+\nu_{m-1}\left(t_{m}(\epsilon)\right)^{m-1}\right)_{m-1}\right. \\
& \left.+l \nu_{m}\left(\left(t_{m}(\epsilon)\right)_{1}\right)^{m} \ln \left(\frac{1}{\epsilon}\right)\right\}^{\frac{1}{m}}
\end{aligned}
$$

where $\nu_{m}$ is defined by equality (5.64), in which the operator $\Psi_{m}$ is defined by (5.51), (5.3) and (4.46).

Proof. First we shall prove assertion (ii). Assume that $m>0$. Let $\mu_{0}^{+}(\lambda)$ be the maximal characteristic branch of the operator $H_{0}$ with respect to $V$ in the gap $(-\infty, 0)$ of $\sigma\left(H_{0}\right)$ and $\tilde{\mu}_{0}(\lambda)$ be the maximal eigenvalue of the finite-rank portion $\Phi(\lambda)$ of the Birman-Schwinger operator $X_{V}(\lambda)$. By Lemma 5.4, $\tilde{\mu}_{0}(\lambda)$ has the asymptotic expansion (5.52) for $\lambda \uparrow 0$. Making use of Proposition 4.8 and the same arguments, as in the proof of Theorem 5.3, we have estimates for some $\bar{S}>0$ and a small enough $\lambda<0$ :

$$
\sigma(\lambda)-\bar{S} \leq \mu_{0}^{+}(\lambda) \leq \sigma(\lambda)+\bar{S}
$$

where the function $\sigma(\lambda)$ is defined on $(-\infty, 0)$ and has the form:

$$
\sigma(\lambda)=\frac{\nu_{0}}{|\lambda|^{\frac{m}{l}}}+\frac{\nu_{1}}{|\lambda|^{\frac{m-1}{l}}}+\frac{\nu_{2}}{|\lambda|^{\frac{m-2}{l}}}+\cdots+\frac{\nu_{m-1}}{|\lambda|^{\frac{1}{l}}}+\nu_{m} \ln \left(\frac{1}{|\lambda|}\right) .
$$

Then, making use of Proposition 3.16, we get the following estimates for the bottom virtual eigenvalue $\lambda_{\mathbf{0}}(\gamma)$ of $H_{\gamma}$ for a small enough $\gamma<0$ :

$$
\Lambda_{+}\left(\frac{1}{|\gamma|}\right) \leq \lambda_{\mathbf{0}}(\gamma) \leq \Lambda_{-}\left(\frac{1}{|\gamma|}\right)
$$

where $\Lambda_{+}$and $\Lambda_{-}$are the inverses of the functions $\sigma(\lambda)+\bar{S}$ and $\sigma(\lambda)-\bar{S}$, respectively. Like in the proof of Theorem 5.3, in order to find the bounds $\Lambda_{+}\left(\frac{1}{|\gamma|}\right)$ and $\Lambda_{-}\left(\frac{1}{|\gamma|}\right)$ in (5.99), we should solve the equation:

$$
t=\epsilon \theta(t)
$$

where

$$
\theta(t)=\left(\nu_{0}+\nu_{1} t+\cdots+\nu_{m-1} t^{m-1}+l \nu_{m} t^{m} \ln \left(\frac{1}{t}\right)+\psi t^{m}\right)^{\frac{1}{m}},
$$

$t=|\lambda|^{\frac{1}{\tau}}, \epsilon=|\gamma|^{\frac{1}{m}}$ and $\psi$ takes one of the values: $\bar{S}$ or $-\bar{S}$. Then, making use of Lemma 5.5 and estimates (5.99), we obtain assertion (ii) of the theorem.

We now turn to assertion (i). Observe that in the case $m=0$ the finite-rank portion $\Phi(\lambda)$ of the Birman-Schwinger operator $X_{V}(\lambda)$ is of rank one and has the form:

$$
\Phi(\lambda)=\ln \left(\frac{1}{|\lambda|}\right) \eta_{\mathbf{0}}\left(\cdot, h_{\mathbf{0}}\right) h_{\mathbf{0}}
$$


The latter operator has only one non-zero branch of eigenvalues:

$$
\mu_{\mathbf{0}}(\lambda)=\ln \left(\frac{1}{|\lambda|}\right) \eta_{\mathbf{0}}\left\|h_{\mathbf{0}}\right\|^{2}
$$

Using the same arguments, as in the proof of assertion (ii), we prove assertion (i). The theorem is proven.

Remark 5.7. The asymptotic formula (5.95)-(5.97) is well known in the case $l=1$ and $d=2$ (see [S], Ch. 7 and [S1]).

Remark 5.8. In $[\mathrm{N}-\mathrm{W}]$ under conditions $2 l>d, \quad 0 \leq V \in L_{1}\left(\mathbb{R}^{d}\right)$ an asymptotic formula for the bottom virtual eigenvalue of the operator $H_{\gamma}$ has been obtained, which has the following form in our notation:

$$
\lambda_{\mathbf{0}}(\gamma)=-|\gamma|^{\frac{2 l}{2 l-d}}\left\{\left(\xi_{\mathbf{0}} \int_{\mathbb{R}^{d}} V(\mathbf{x}) d \mathbf{x}\right)^{\frac{2 l}{2 l-d}}+o(1)\right\} \text { for } \gamma \uparrow 0 .
$$

We see that the remainder of this asymptotic formula has the order $o\left(|\gamma|^{\frac{2 l}{2 l-d}}\right)$. On the other hand, we see that the remainder of the asymptotic expansions (5.40) and (5.98) has the order $O\left(|\gamma|^{\frac{4 l-d}{2 l-d}}\right)$. This means that for $2 l>d$ Theorems 5.3 and 5.6 yield more precise asymptotic representations of the bottom virtual eigenvalue of the operator $H_{\gamma}$ than the asymptotic representation (5.100) of [N-W].

\section{References}

[Ar-Z] J. Arazy and L. Zelenko, Finite-dimensional perturbations of self-adjoint operators, Integral Equations and Operator Theory, 34 (1999), 127-164.

[Ar-Z2] J. Arazy and L. Zelenko, Virtual eigenvalues of the high order Schrödinger operator II, to appear in Integral Equations and Operator Theory.

[Bau] H. Baumgärtel, Analytic Perturbation Theory for Matrices and Operators, Birkhäuser Verlag Basel-Boston-Stuttgart, 1985.

[Bi1] M. Sh. Birman, The spectrum of singular boundary problems, (Russian) Mat. Sb. (M.S.) 55 (97) (1961), 125-174, (English) Amer. Math. Soc. Transl. 53 (1966), 23-80.

[Bi2] M. Sh. Birman, Discrete Spectrum in the Gaps of a Continuous One for Perturbations with Large Coupling Constant, Advances in Soviet Mathematics, vol. 7 (1991), 57-73, Amer. Math. Soc., Providence, Rhode Island.

[Bi3] M. Sh. Birman, On the Number of Eigenvalues in a Quantum Scattering Problem, Vest. LSU 16 (1961), No 3, 163-166.

[Bi-So] M. Sh. Birman and M. Z. Solomyak, Estimates for the Number of Negative Eigenvalues of the Schrödinger Operator and its Generalizations, Advances in Soviet Mathematics, vol. 7 (1991), 1-55, Amer. Math. Soc., Providence, Rhode Island. 
[B-G-S] R. Blankenberg, M.L. Goldberger and B. Simon, The Bound State of Weaklycoupled Long-range One-dimensional Quantum Hamiltonians, Ann. Phys. 108 (1977), 69-78.

[Gl] I. M. Glazman, Direct Methods of Qualitative Spectral Analysis of Singular Differential Operators, I.P.S.T., Jerusalem, 1965.

[Kl] M. Klaus, On the Bound State of Schrödinger operators in One-Dimension, Ann. Phys. 108 (1977), 288-300.

[L-Thr] E. Lieb and W. Thirring, Inequalities for the moments of the eigenvalues of the Schrödinger Hamiltonian and their relation to Sobolev inequalities, Studies in Math. Phys., Essays in Honor of Valentine Bargman, Princeton, 1976 .

$[\mathrm{N}-\mathrm{W}] \quad \mathrm{Y}$. Netrusov and T. Weidl, On Lieb-Thirring Inequalities for Higher Order Operators with Critical and Subcritical Powers, Comm. Math. Phys. 182 (1996), No 2, 355-370.

[Re-Si] M. Reed and B. Simon, Methods of Modern Mathematical Physics, IV: Analysis of Operators, Academic Press, New York, 1978.

[Ri-Nag] F. Riesz et B. Sz-Nagy, Lecons D'Analyse Functionnele, Akademiai Kiado, Budapest, 1972.

[Sc] Y. Schwinger, On the bound states for a given potential, Proc. Nat. Acad. Sci. U.S.A. 47 (1961), 122-129.

[S] B. Simon, Trace Ideals and their Applications, Cambridge University Press, London-New York-Melbourne, 1979.

[S1] B. Simon, The Bound State of Weakly Coupled Schrödinger Operators in One and Two Dimensions, Ann. Phys. 97 (1976), 279-288.

[Sob] A. V. Sobolev, Weil Asymptotics for the Discrete Spectrum of the Perturbed Hill Operator, Advances in Soviet Mathematics, vol. 7 (1991), 159-178, Amer. Math. Soc., Providence, Rhode Island.

[W] T. Weidl, Remarks on virtual bound states of semi-bounded operators, Comm. Partial Differential Equations 24 (1999), No 1-2, 25-60.

Jonathan Arazy

Department of Mathematics

University of Haifa

31905 Haifa, Israel

E-mail address:

jarazy@math.haifa.ac.il
Leonid Zelenko

Department of Mathematics

University of Haifa

31905 Haifa, Israel

E-mail address:

zelenko@math.haifa.ac.il 